\title{
A Novel Precast Concrete Beam-Column Connection With Replaceable Energy-Dissipation Connector: Experimental Investigation and Theoretical Analysis
}

Chao Tong

Southeast University

Jing Wu ( $\sim$ seuwj@seu.edu.cn )

Southeast University

Chunyu Li

Shijiazhuang Tiedao University

\section{Research Article}

Keywords: precast concrete beam-column connection, additional bending moment, replaceable energydissipation connector, energy-dissipation capacity, load capacity

Posted Date: February 23rd, 2021

DOl: https://doi.org/10.21203/rs.3.rs-219155/v1

License: (c) (1) This work is licensed under a Creative Commons Attribution 4.0 International License.

Read Full License 
1 A novel precast concrete beam-column connection with replaceable theoretical analysis

1 The Key Laboratory on Concrete and Prestressed Concrete Structures of Ministry of Education of China,

${ }_{2}$ School of Civil Engineering, Shijiazhuang Tiedao University, Shijiazhuang 050043, China bending moment produced by the combined action of the pressure generated by the concrete compression zone and the tension generated by the reinforcements needs to be reduced. A novel precast concrete beam-column connection is proposed herein. In the proposed connection, the precast beam is laid on a steel corbel embedded in the precast column. A novel replaceable energydissipation connector (REDC) is placed at the bottom of the steel corbel, which ensures that it is in the same horizontal position aligned with the longitudinal reinforcement at the bottom of the connection. In addition, there is a narrow vertical slot adjacent to the column face. The total yield capacity of the top reinforcement is larger than that of the bottom REDC energy-dissipation connector. Theory study focused on the structural features and mechanical mechanism of this novel precast connection. Three low-cycle quasi-static loading tests were carried out on a single full- 
1 scaled specimen by replacing three REDCs with different sizes. The cracking pattern of this novel

2 precast connection, and the effects of different parameters of the REDCs on the energy-dissipation

3 capacity and load capacity of the connections were discussed. By performing a finite-element

4 simulation, a method for reducing the additional bending moment and keeping the top

5 reinforcements always in elastic was developed. In addition, the relevant design suggestions were

6 provided. The conclusion shows that the seismic performance of this novel precast concrete beam-

7 column connection is excellent. The positive and negative bending moments of novel precast

8 concrete beam-column connection both reduce the additional bending moment. In view of this,

9 the bending moment of the novel connection is a controllable variable.

10 Keywords: precast concrete beam-column connection; additional bending moment; replaceable

11 energy-dissipation connector; energy-dissipation capacity; load capacity.

\section{Introduction} gradually replaced the traditional cast-in-place structure. It promotes the process of construction

16 industrialization and satisfies the needs of the future development of the construction industry

17 (Hassoun, M. N, and Al-Manaseer, A. 2020). Although the "wet" fabricated structures can be

18 equivalent to or close to the cast-in-place structures, it inherits the characteristics of damage

19 dispersion of the cast-in-place structures under strong earthquakes (Liu H, Yan Q, Du X. 2017).

20 Under seismic action, the ductility of the plastic hinge at the beam end of the structures mainly

21 depends on the yield of the longitudinal reinforcements at the beam end. Because of the crushing 
of concrete in the compression zone and the buckling of the longitudinal reinforcements caused by the rotation of the plastic hinge, the moment capacity decreases rapidly. This type of failure occurs particularly easily under the negative moment, because the height of the compression zone in the design philosophy. The significantly increase of the beam overstrength moments and hence column mainly due to the large area of the slab reinforcement in the flange of the frame beam, which contributes to the bending moment resistance together with the longitudinal reinforcement in the that must be solved for prefabricated concrete structures, particularly for "wet" prefabricated concrete structures, which are equivalent to cast-in-place structures. The main reason for this reinforcement, which contributes to the moment capacity together with the longitudinal 
moments, which can result in hinging of columns and the formation of an undesirable soft-story mechanism. These issues affect the structural safety (Huang et al. 2020).

In recent decades, the seismic performance of fabricated structures has been studied deeply and comprehensively. In the 1970s, Blakeley, Park (1971), and Thompson (1975) experimentally investigated the joints and structures of prefabricated frames to understand their behavior under cyclic load reversals. The test results indicated that prefabricated concrete structures have favorable integrity, ductility properties, and deformation recovery capabilities. The Precast Seismic Structural System Research Program (PRESSS) began in 1991 and was jointly undertaken by researchers in the United States and Japan. As one of the first research projects in this field, the PRESSS involved a detailed investigation of precast concrete structures (Priestley, M J Nigel. 1991; Priestley, M J Nigel.1996; Priestley, M J Nigel., and Macrae G A. 1996; Vasconez R M., Naaman A E, Wight J K. 1998). In 2003, the European Union initiated a seismic research project for precast concrete structures based on Eurocode 8 (Fan Li., Lv Xilin, Zhao Bin. 2007), in which the seismic performance of beam-column connections and frames was investigated, and useful results were obtained. Nakaki et al. (1994) proposed precast concrete beam-column connections with dywidag ductile connector (DDC). Englekirk (1995) tested precast concrete beam-column connections with DDC ductile connectors under quasi-static cyclic loading to evaluate their seismic performance. Under $3.5 \%$ interstory drift, the connections and components exhibited no obvious damage. Englekirk (1996) first used the DDC ductile connector in the Wiltern Center Parking in Los Angeles. This structure has been analyzed by Englekirk (2002) and applied to famous buildings, including the Highland Center in Hollywood and the Paramount apartment in San Francisco. Bara 
1 Chang et al. $(2012,2014)$ tested full-scale and scaled specimens of frames with DDC. The results confirmed that the connections with the DDC had a stable energy-dissipation capacity and that the seismic performance of the DDC frames was better than that of conventional frames. Kenyon et al. (2008) conducted a finite-element simulation to evaluate the seismic performance of precast concrete frames with a DDC.

Additionally, according to the Damage Avoidance Design (DAD) approach (Bacht T et al. 2011; Mander J B, Cheng C-T. 1997; Mander T J et al. 2009; Linjie Huang et al. 2020), various yielding steel devices have been proposed and used in structures. Damage avoidance can be achieved in moment frames by using special devices that do not undergo degradation of the dissipative performance with use. Compared with the conventional moment frames and "wet" precast frames, precast frames designed according to the DAD approach. This principle reduces the amount of frame repair needed, and the minimized disruption substantially reduces the economic and business impacts. Amaris et al. (2008) and Marriott (2009) proposed a tension and compression yield energy-dissipation device. However, this type of energy-dissipation device is installed outside the beams; thus, the transfer force is indirect and easily causes the failure of the anchoring of the conversion block. Chanchi et al. $(2018,2019)$ tested and simulated an asymmetric friction energy-dissipation device and applied it to steel structures. Rodgers (2019) tested a series of lead squeeze dampers. Oh et al. (2009) and Koken et al. (2013) have tested full-scale specimens of steel beam-column connections with steel slit dampers and compared them with the conventional steel connections. The test results indicated that the steel slit damper provides a reliable energy-dissipation capacity. Chi et al. (2012) experimentally and analytically investigated 
the cyclic response of a post-tensioned column base connection with a buckling-restrained steel plate (BRS). The results indicated that the BRS plate had good energy-dissipation capacity when yielding under tension and compression. These steel energy-dissipation devices have an excellent and stable energy-dissipation capacity but have disadvantages; e.g., they have a complex structure, require extra space, and are difficult to replace. significant seismic event, a plastic hinge can lengthen by $2 \%-5 \%$ of the beam depth (Fenwick R increase the lateral resistance of a moment resisting frame and cause significant damage to the floor diaphragm (Bull. D. K. 2003; Peng et al. 2009). To solve this problem, Ohkubo et al. (2009) proposed a slotted beam-column connection and performed experiments to examine the shear transfer mechanism in the beam-end region. The results indicated that the slotted beams have good flexural characteristics. Ohkubo et al. (2004) tested a reinforced-concrete (RC) slotted-beam cracks and damage in the floor slab for slotted-beam frames were significantly reduced compared with those for the conventional frames. Au (2010), J.D.R. Byrne et al. (2012), and C. A. Muir et al. (2013) tested slotted-beam connections and frames and verified that the slotted-beam structures beam connections to examine the effects of the vertical slot position on the seismic performance and damage to the connection. Many studies have been performed on slotted-beam connections in 
cast-in-place concrete structures, but there have been relatively few studies on slotted-beam connections in precast concrete structures. At present, the research on slotted-beam connections mainly focuses on cast-in-place concrete frame structures, and there is little research on the fabricated frame structures. concrete connection with a replaceable energy-dissipating connector (REDC). At the same horizontal position as the longitudinal reinforcement at the bottom of the beam, the REDC energydissipation connector is connected to the longitudinal reinforcements in the beam and column by facilitate construction of the beam and column assembly. The vertical slot is installed in the bottom capacity ratio of the top reinforcement to the bottom REDC energy-dissipation connector is increased and the height of the concrete compression zone is reduced, the neutral axis of the connection will move upward, and the additional bending moment of the beam-column connection will reduce. At the same time, when the frame composed by these novel connections is subjected to earthquake ground motions, the damage to the structure is mainly concentrated in the REDCs. After the earthquake, the structure can be repaired quickly by replacing the REDCs. Because of the reducing of the additional bending moment, the moment capacity of the connection is

19 controlled by the REDCs as much as possible, and the principle of "strong column weak beam" is easily realized. Therefore, the principles of "strong shear weak moment" and "strong connection weak member" can be achieved. In this study, the seismic performance of the novel precast 
1 connection was investigated via a theoretical analysis and experimental research, and design values

2 were recommended for the relevant parameters.

\section{2. Novel precast concrete beam-column connection with REDC}

\subsection{Connection overview}

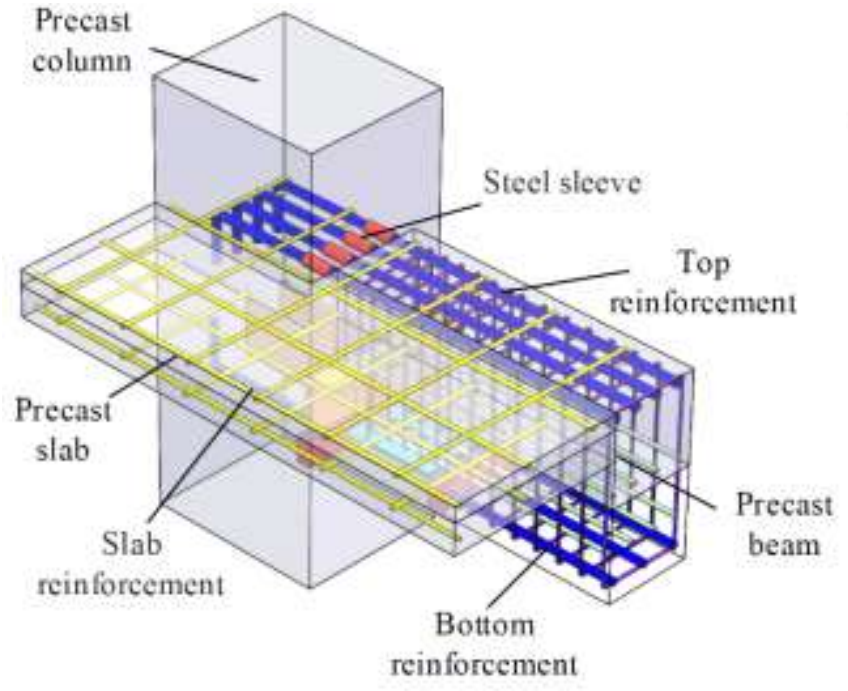

(a) Side elevation (from top)

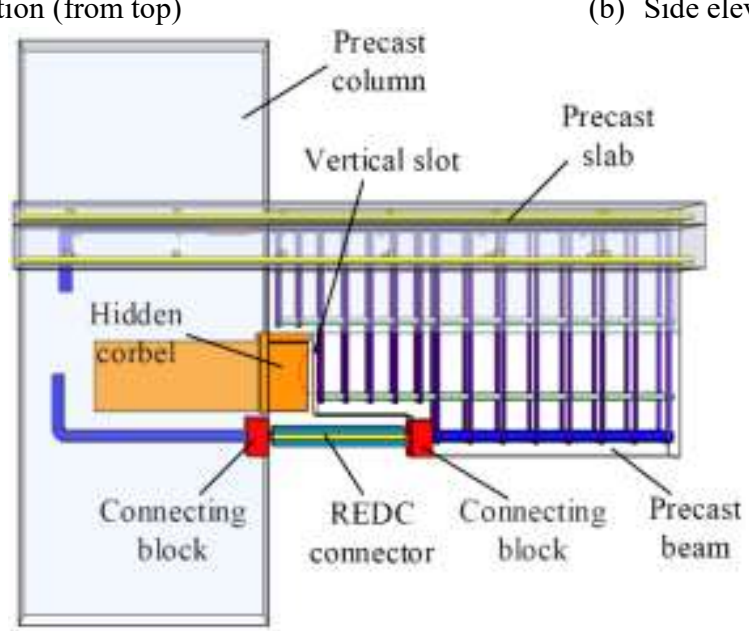

(c) Front elevation

Fig 1 Description of the novel precast concrete beam-column connections with the REDC

A schematic configuration of the novel precast connection assembly is presented in Fig. 1.

The connection is composed of four main parts: the precast concrete beam, the precast concrete column, the REDC, and the hidden corbel. As shown in Fig. 1(a), the top longitudinal 
1 reinforcements (containing slab reinforcements) exceeds the area of the REDC core plate. As

2 shown in Fig. 1(b), the hidden corbel is embedded in the column and lapped with the rabbet of the

3 precast beam. The $\pi$-shaped hidden corbel consists of two welded steel plates, which contribute to

4 the shear and bending capacities of the corbel. At the same horizontal position as the longitudinal

5 reinforcement at the bottom of the beam, the REDC energy-dissipation connector is connected to

6 the longitudinal reinforcement in the beam and column by connecting blocks. As shown in Fig.

7 1(c), the vertical slot is installed at the bottom of the beam, immediately adjacent to the column

8 face.

$9 \quad$ 2.2.Details of REDC

10

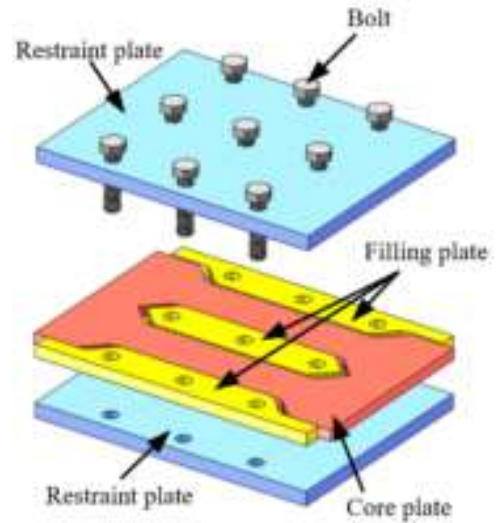

(a) Details of REDC

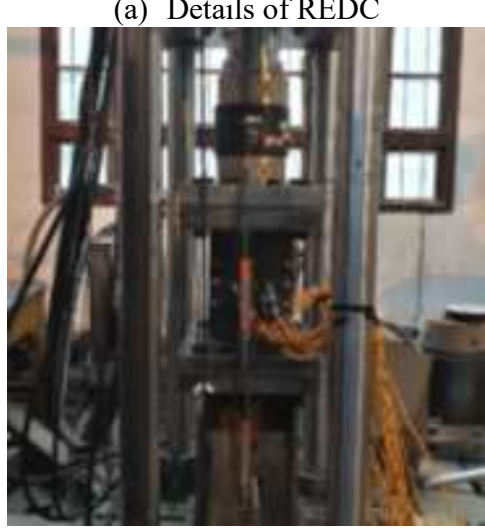

(c) Test of REDC

Fig 2 Details and test of REDC

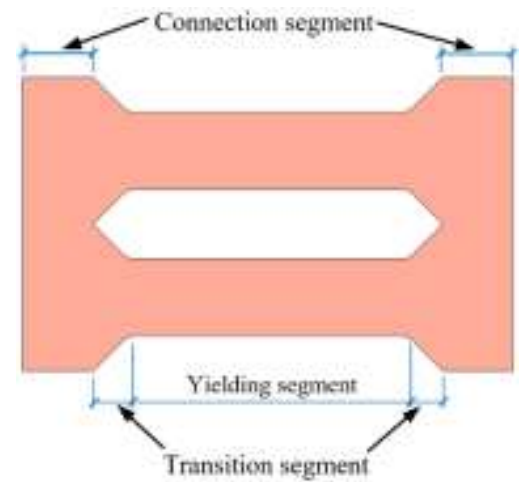

(b) Details of REDC core plate

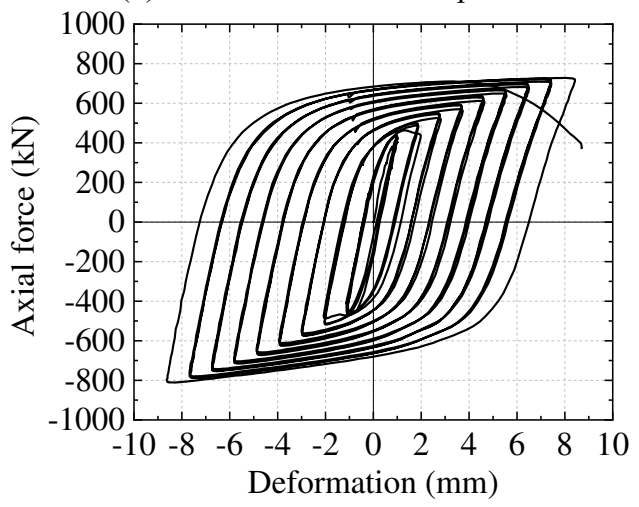

(d) Hysteric curve of REDC

The REDC is a buckling-restrained energy dissipater, and proposed by Xie (2020). In addition, 
1 Li (2020) proposed a precast concrete frame with replaceable energy-dissipating connectors

2 (REDC-PCF) and test it. Figs.2 (a)-(d) are details and test of REDC. The REDC is assembled by

3 bolts and composed of a core plate, filling plate and restraint plate (Fig. 2 (a)). The core plate is

4 welded to structural members and yields under repeated axial loads. The filling plate and restraint

5 plate are used to avoid the compression buckling of the core plate. The section of core plate varies

6 along the length (Fig. 2 (b)), and it is divided into yielding segment, transition segment, and

7 connection segment. In addition, the stable hysteretic energy dissipation capability of REDC

8 observed in an axial cyclic tension-compression test can be observed from Figs. 2 (c) and (d) (Xie.

9 2020). The damage to the connection is mainly concentrated on the REDC. The energy-dissipation

10 capacity of the novel precast connection is provided by the tension and compression plastic

11 deformation of the REDC. After the earthquake, the structures can be repaired quickly by replacing

12 the REDCs.

$13 \quad$ 2.3.Mechanics of the novel precast connection

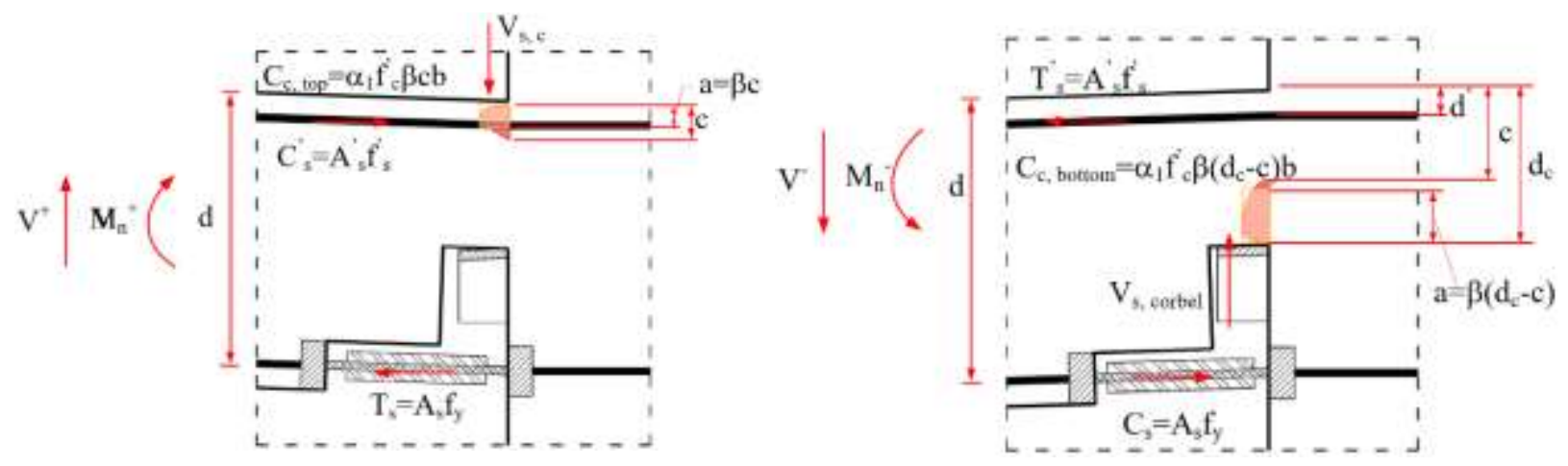

(a) Positive moment (gap closing)

(b) Negative moment (gap opening)

Fig. 3 Force distribution in the connection under positive and negative bending

Figs. 3(a) and (b) illustrate the forces in this novel precast connection at positive (gap closing) 
respectively.

The flexural response of this novel precast connection is governed by the yielding of the REDC core plate and the concrete compression zone. The positive nominal flexural strength $\left(\mathrm{M}_{\mathrm{n}}{ }^{+}\right)$ can be calculated similarly to that in the case of a conventional RC connection, i.e., by multiplying the REDC yield force about a lever arm, as indicated by Eq. (1). This is an approximation that conservatively ignores the force in the top reinforcement and any additional confinement to the concrete hinge from the column face.

$$
\mathrm{M}_{\mathrm{n}}^{+}=\mathrm{A}_{\mathrm{s}} \mathrm{f}_{\mathrm{y}}(\mathrm{d}-\mathrm{a} / 2)
$$

where $A_{s}$ represents the equivalent cross-sectional area of the REDC core plate; $f_{y}$ represents the yield strength of the REDC core plate; $d$ represents the beam effective depth; a represents the Whitney equivalent rectangular concrete stress block depth.

The flexural mechanism for negative moments in this novel precast connection differs from that for conventional RC beams. There is no concrete compression at the beam soffit, owing to the slot. However, there is concrete compression at the bottom of the concrete top-hinge, as shown in Fig. 2(b). The negative nominal flexural strength can be accurately approximated as follows:

$$
M_{n}{ }^{-}=A_{s} f_{y}\left(d-d^{\prime}\right)+a_{1} f^{\prime} \beta\left(d_{c}-c\right) b\left(d_{c}-a / 2\right)
$$

where d' represents the depth to the top reinforcement; $a_{1}$ and $\beta$ are concrete stress block factors; $\mathrm{f}_{\mathrm{c}}$ represents the specified 28 - $\mathrm{d}$ concrete compressive strength; $d_{c}$ represents the depth of the concrete top-hinge in this novel precast connection; c represents the neutral-axis depth (measured from the top surface of the beam); b represents the section width of the beam.

For gap-opening rotations (positive moment), the shear forces transferred through the 
2 in China (GB50010-2010) (2010), as follows:

$$
\mathrm{V}^{+} \leq \mathrm{V}_{\mathrm{s}, \mathrm{c}}=0.25 \beta_{\mathrm{c}} \mathrm{f}_{\mathrm{c}}^{\prime} \mathrm{bh}_{0}
$$

where $V_{s,}$ is the shear forces transferred through the compression concrete zone; $\beta_{\mathrm{c}}$ is the coefficient of concrete strength ( $=1.0$ for C50 concrete), and $\mathrm{h}_{0}$ represents the effective height of the cross section ( $h_{0}$ represents the $d_{c}$ in this novel precast connection).

For gap-closing rotations (positive moment), the shear forces transferred via the hidden corbel can be designed using Eq. (3).

$$
\mathrm{V}^{-} \leq \mathrm{V}_{\mathrm{s}, \text { corbel }}
$$

where $\mathrm{V}_{\mathrm{s} \text {, corbel }}$ represents the shear capacity provided by the hidden corbel.

\section{Experimental study on the novel precast connection}

\subsection{Test specimen}

To experimentally investigate the hysteretic behavior of the novel precast connection under cyclic loading, a full-scale test specimen was fabricated, as shown in Fig. 3. This specimen corresponded to the exterior beam-column connection from a prototype concrete frame in China. According to the Chinese Code for Seismic Design of Buildings (GB50010-2010), this frame being designed is located at the area of seismic intensity 8 , seismic group 1 , and soil type II, with a peak ground acceleration (PGA) of $0.4 \mathrm{~g}$ for the maximum considered earthquake (MCE) $(2 \%$ exceedance probability in 50 years). As shown in Fig. 4(a), the column was $2800 \mathrm{~mm}$ tall, with a cross section having dimensions of $500 \mathrm{~mm} \times 700 \mathrm{~mm}$. The beam was 1,800 $\mathrm{mm}$ long, with a cross section of $300 \mathrm{~mm} \times 600 \mathrm{~mm}$. The cross sections and reinforcement layouts of the beam and 
column are shown in Figs. 4(b)-(e). The REDC was connected by the core plate, the restraining plates, and the filler plates using bolts. The dimensions and parameters of the REDCs are shown in Figs. 4(f)-(i). There were 1-mm gaps between the filler plates, restraining plates, and core plate. There was a sufficient gap between the filler plates and the core plate to satisfy the compression deformation of the core plate. Au (2010) proposed that when $\mathrm{A}^{\prime}{ }_{\mathrm{s}} \mathrm{f}_{\mathrm{y}} / \mathrm{A}_{\mathrm{s}} \mathrm{f}_{\mathrm{y}}>2.5$, the neutral axis of the connection will move upward, and the top reinforcement to remain elastic. Therefore, for the top longitudinal reinforcement in this novel precast connection, six 28-mm-diameter deformed bars and HPB400 steel (yield stress of $400 \mathrm{MPa}$ ) were used to decrease the yield-capacity ratio of noting that these six bars include the equivalent bars in the slabs. Therefore, the flange effect of slabs in connection was considered by equivalent reinforcement. In addition, the thickness of the simplify the construction of the specimen, the sleeve-connected top reinforcements were replaced with continuous reinforcements. One $\pi$-shaped steel hidden corbel was embedded in the column, at the half-height of the beam. The length of the corbel was $80 \mathrm{~mm}$, and the cross section of the flange plate in the $\pi$-shaped steel corbel was $300 \mathrm{~mm} \times 18 \mathrm{~mm}$. The cross section of the web plates $\mathrm{mm}$ wide, which was sufficient to accommodate $4.0 \%$ drifts without contact between the beam bottom of the beam) was $300 \mathrm{~mm}$. 


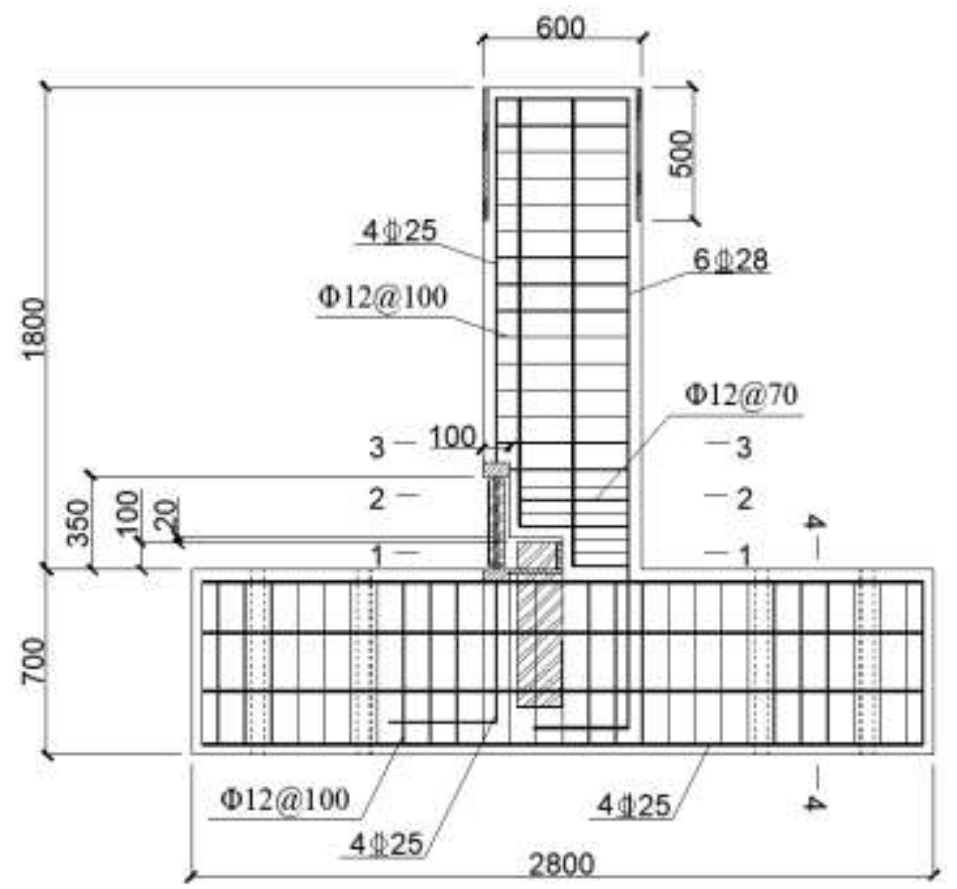

(a) Specimen

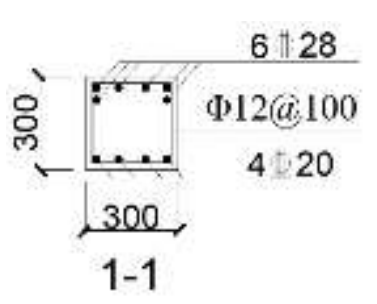

(b) Beam section 1-1

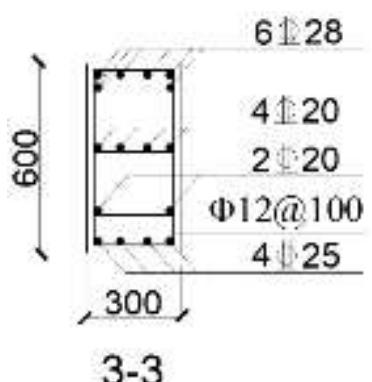

(d) Beam section 3-3

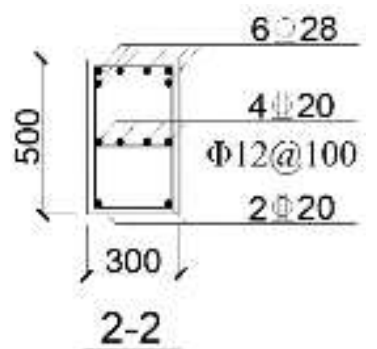

(c) Beam section 2-2

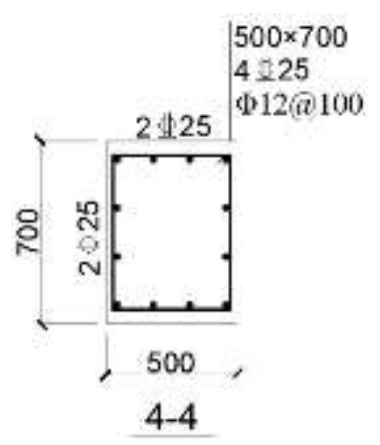

(e) Column section 4-4

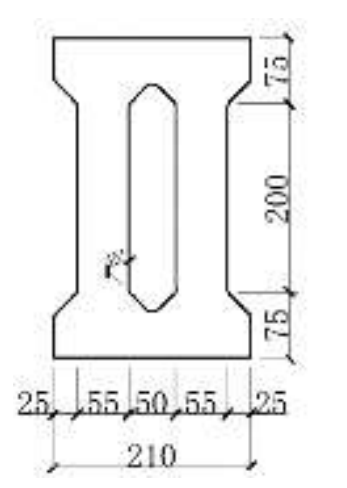

(f) Core plate $(12 / 10 \mathrm{~mm})$

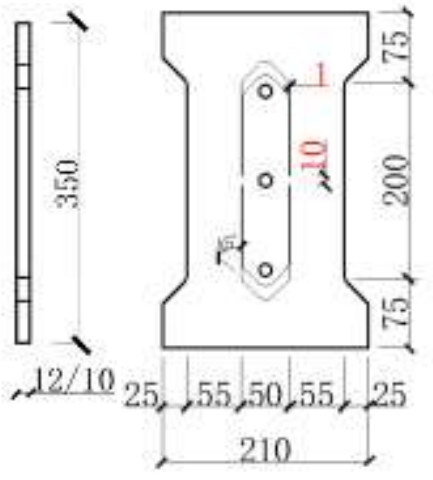

(g) Core plate $(14 \mathrm{~mm})$

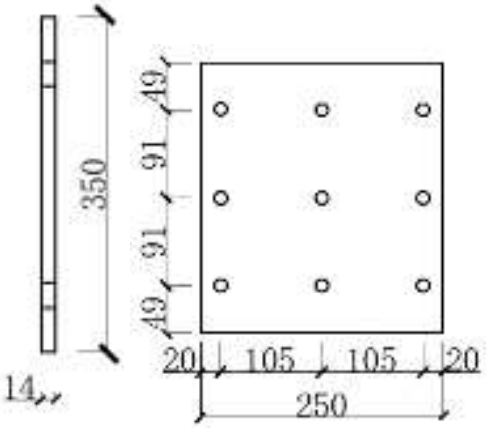

(h) Restraining plate

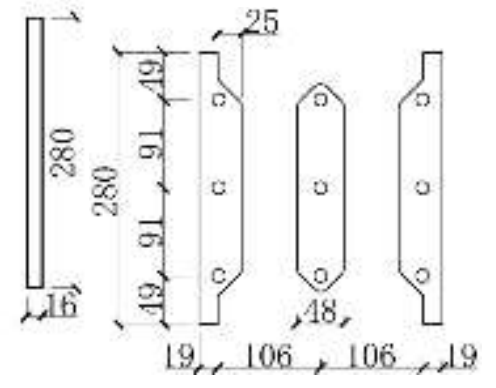

(i) Filler plate

Fig. 4 Details of the tested connections (all dimensions have units of $\mathrm{mm}$ )

5

6

\subsection{Material properties}

For the test specimen, the material properties of the reinforcements, the $\pi$-shaped steel corbel 
1 and connecting blocks are presented in Table 1.

Table 1 Mechanical properties of the structural steels in the specimen

\begin{tabular}{|c|c|c|c|c|}
\hline Material & Structural components & $\begin{array}{l}\text { Yielding strength } \\
f_{\mathrm{y}}(\mathrm{MPa})\end{array}$ & $\begin{array}{l}\text { Ultimate strength } \\
\qquad f_{\mathrm{p}}(\mathrm{MPa})\end{array}$ & $\begin{array}{l}\text { Young's modulus } \\
\qquad E_{\mathrm{s}}\left(\mathrm{N} / \mathrm{mm}^{2}\right)\end{array}$ \\
\hline Q345 & Steel corbel and connecting blocks & 443.98 & 586.32 & 211023 \\
\hline HRB400 & Reinforcement D28 & 420.18 & 490.04 & 206734 \\
\hline HRB400 & Reinforcement D25 & 417.21 & 478.92 & 206837 \\
\hline HRB400 & Stirrup 14 & 476.12 & 527.33 & 210322 \\
\hline
\end{tabular}

The grade of the concrete in the specimen was C50. Three groups of compressive-strength tests were performed, and the results are presented in Table 2. The test results all satisfied the GB500102010.

Table 2 Cube strength of C50 concrete

\begin{tabular}{ccccc}
\hline Test specimen & 1 & 2 & 3 & Average \\
\hline Strength $(\mathrm{MPa})$ & 60.22 & 51.38 & 53.47 & 55.02 \\
\hline
\end{tabular}
The REDC was fabricated using the Q235 plate. Three samples of the Q235 plate were tested, and the results are presented in Table 3. In addition, the carbon dioxide gas shielded arc welding is used to weld the core plate and the connecting blocks, and the type of welding wire is ER50-6.

Table 3 Material test results for Q235 steel

\begin{tabular}{cccccc}
\hline No. & $\begin{array}{c}\text { Yielding strength } \\
f_{\mathrm{y}}(\mathrm{MPa})\end{array}$ & $\begin{array}{c}\text { Ultimate strength } \\
f_{\mathrm{p}}(\mathrm{MPa})\end{array}$ & $\begin{array}{c}\text { Young's } \\
\text { modulus } \\
E_{\mathrm{s}}\left(\mathrm{N} / \mathrm{mm}^{2}\right)\end{array}$ & $\begin{array}{c}\text { Yield ratio } \\
(\%)\end{array}$ & $\begin{array}{c}\text { Ductility } \\
(\%)\end{array}$ \\
\hline A & 271.29 & 458.43 & 211468 & 59.18 & 38.51 \\
B & 262.31 & 456.30 & 214452 & 57.49 & 39.42 \\
C & 270.26 & 460.65 & 212122 & 58.67 & 39.55 \\
Average & 267.95 & 458.46 & 212681 & 58.45 & 39.16 \\
\hline
\end{tabular}

\section{$11 \quad$ 3.3.Test setup and loading protocol}

The beam-column assembly was rotated $90^{\circ}$ for the test, and the test setup is shown in Fig.

13 5. A lateral load was applied near the beam end using an actuator with a scale of $\pm 1000 \mathrm{kN}$. A

14 displacement transducer with a scale of $\pm 150 \mathrm{~mm}$ was installed at the bottom of the beam to record 
1 and calculate the relative rotation between the beam and the column during the tests. At the ends

2 of the column, two jacks were used to apply an axial load, which corresponded to an axial

3 compression ratio of 0.2, as recommended by the Code for Seismic Design of Buildings in China

4 (GB50010-2011) (2010), and restrict the horizontal movement of the assembly. Additionally, two

5 displacement transducers were placed at the same position to detect horizontal movement for

6 enhancing the accuracy of the measurements.

The drift sequence in Fig. 6 was adopted in accordance with the American Concrete Institute

8 (ACI) acceptance criteria for moment frames (ACI Committee 374, 2005). The test specimens

9 were subjected to three cycles at drift levels of $0.1 \%, 0.2 \%, 0.5 \%, 0.75 \%, 1.0 \%, 1.5 \%, 2.0 \%, 2.5 \%$,

11 for closing the gaps between the aggregates in the concrete.

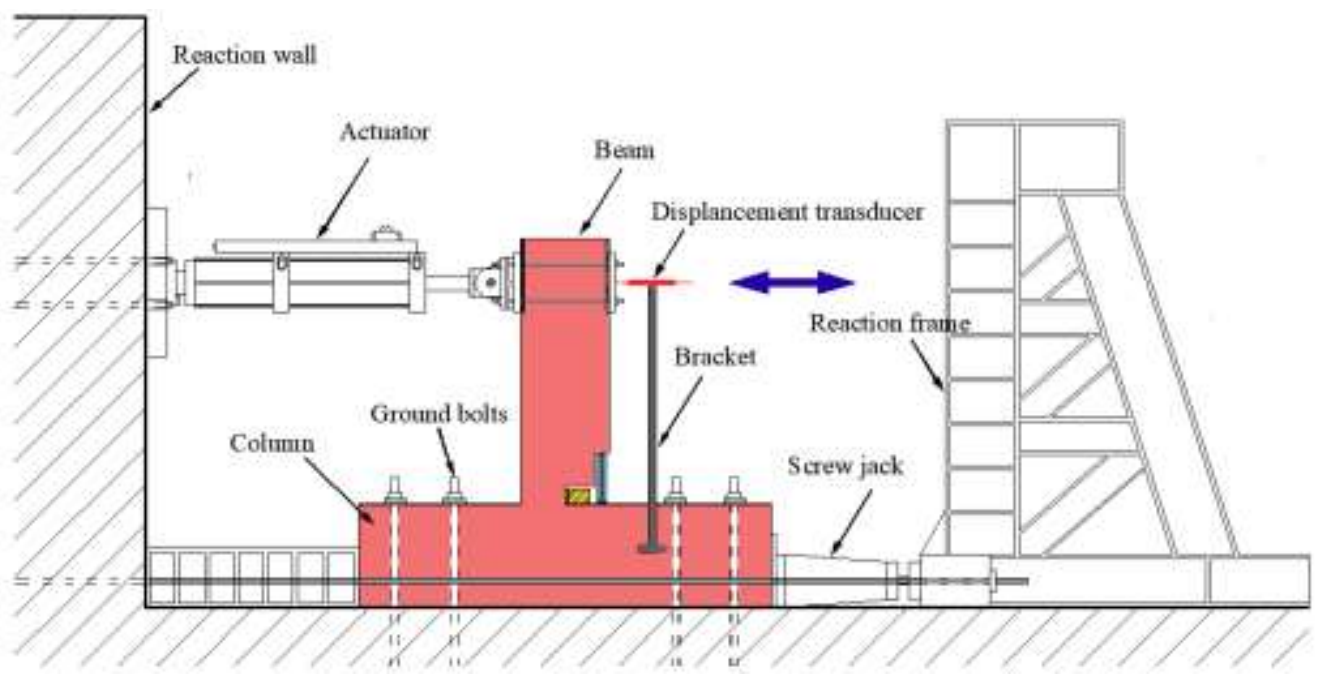

(a). Test setup and instrumentations 


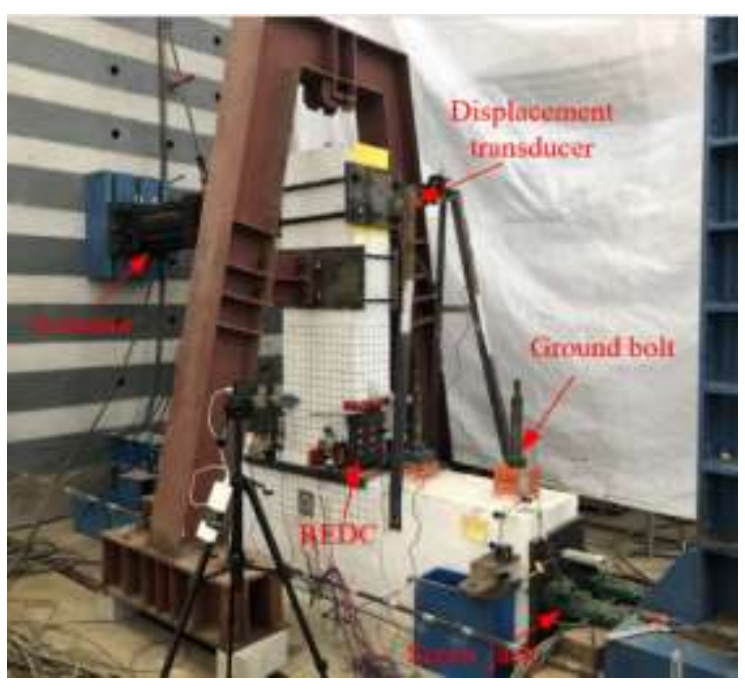

(b). Photograph of the specimen under test

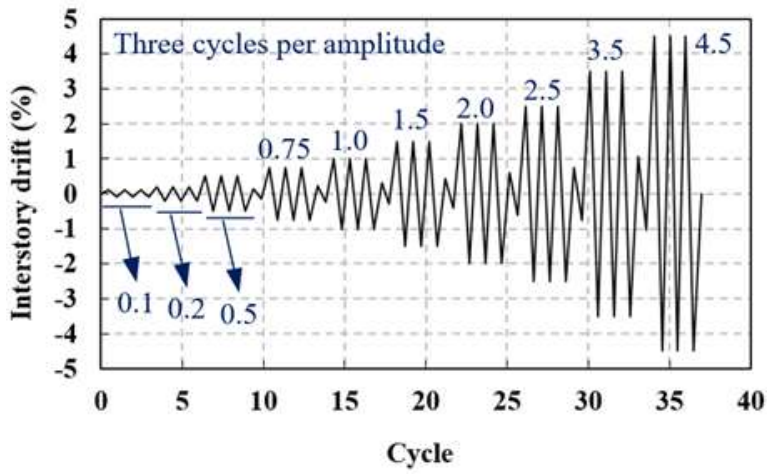

Fig. 6 Quasi-static loading

Fig. 5 The specimen under test

\subsection{Test matrix}

The thickness of the REDC core plate and the structural details of the REDC are significant design parameters that affect the hysteretic responses of this novel precast connection. To investigate the seismic behaviors in this novel precast connection, a specimen was subjected to three tests with variations in the design parameters. The design parameters used for the tests are presented in Table 4. In Test 1, the thickness of the REDC core plate was $12 \mathrm{~mm}$, and the core plate contained no stoppers and unbonding material. Additionally, 4.8-grade M12 × $60 \mathrm{~mm}$ ordinary bolts were used to connect the REDC. In Test 2, the thickness of the REDC core plate was $10 \mathrm{~mm}$, and the core plate contained no stoppers and unbonding material. Different from the Test 1, 10.9-grade M14 × $60 \mathrm{~mm}$ high-strength bolts were used to connect the REDC in Test 2. In Test 3, the thickness of the REDC core plate was $14 \mathrm{~mm}$, and the connected bolts were 10.9-grade M14 $\times 60 \mathrm{~mm}$ high-strength bolts. Different from Tests 1 and 2, the core plate had stoppers (two 10-mm-long connections) and unbonding material in Test 3 . The stopper in the Test 3 is used to 
1 fix the restraining plates and the filler plates in the center of the core plate, so as to reduce the

2 friction of the core plate and the restraining plates. At the same time, the unbonding material is

3 used to reduce the friction between the core plate and the restraining plates. By reducing friction,

4 the neck phenomenon of the core plate and the stress concentration will be reduced.

5

Table 4 Test matrix

\begin{tabular}{|c|c|c|c|c|c|}
\hline \multirow{2}{*}{$\begin{array}{c}\text { Test } \\
\text { number }\end{array}$} & \multirow{2}{*}{$\begin{array}{l}\text { Thickness of the core plate } \\
\qquad(\mathrm{mm})\end{array}$} & \multicolumn{2}{|c|}{ Bolt parameters } & \multirow{2}{*}{ Stopper } & \multirow{2}{*}{$\begin{array}{c}\text { Unbonding } \\
\text { Material }\end{array}$} \\
\hline & & Type & Size $(\mathrm{mm})$ & & \\
\hline Test 1 & 12 & 4.8-grade & $\mathrm{M} 12 \times 60$ & - & $\mathrm{N}$ \\
\hline Test 2 & 10 & 10.9-grade & $\mathrm{M} 14 \times 60$ & - & $\mathrm{N}$ \\
\hline Test 3 & 14 & 10.9-grade & $\mathrm{M} 14 \times 60$ & $10 \mathrm{~mm}$ & $\mathrm{Y}$ \\
\hline
\end{tabular}

6

\section{Experimental results and discussion}

\subsection{Hysteretic response}

The hysteretic responses of the three tests are shown in Fig. 7. In all the tests, failure occurred via the fracture of the REDC core plate owing to the low-cyclic fatigue. The drift ratios were

11 calculated by dividing the displacement at the tip of the beam by the shear span (the shear span was $1500 \mathrm{~mm}$ ). The drift ratio in the hysteretic response (Fig. 7) was the relative drift of the beam and column, which is measured by the displacement transducer fixed on the column (shows in ratio in the hysteretic curve is smaller than that in loading protocol, due to the gap between the actuator and the specimen and the slight slip in the loading process. In addition, as shown in Fig. 7(a), the positive and negative drifts of Test 1 differed at the same loading level. At the same time, 
1 protocol. It is attributed the rigid-body rotation and slippage of the specimen in the negative drift caused by anchor failure of the two ground bolts in the left side of the specimen. In order to avoid this phenomenon happening again in the Test 2 and Test 3, two anchor failed ground bolts were refastened after the Test 1 , and a stronger torsional moment was applied to the ground bolts.

In the test 1, as shown in Fig. 7(a), the maximum positive drift sustained in Test 1 was 3.41\% (the corresponding drift level in the loading protocol was 3.5\%), and the maximum positive force was $225 \mathrm{kN}$ corresponding the first cycle at this level. The negative maximum drift was $-2.69 \%$ (the corresponding drift level in the loading protocol was $-3.5 \%$ ), and the maximum negative forces was $-373.9 \mathrm{kN}$ corresponding the first cycle at this level. One side of the REDC core plate fractured at $-1.03 \%$ drift (during the second $-3.5 \%$ drift cycle corresponding to the loading protocol). In addition, the REDC core plate buckled at $-0.21 \%$ drift (during the third $-3.5 \%$ drift cycle corresponding to the loading protocol), because the ordinary bolts connected the restraining plates and the filler plates were fractured.

In the test 2, as shown in Fig. 7(b), the positive maximum drift sustained in Test 2 was 2.24\% (the corresponding drift level in the loading protocol was $2.5 \%$ ), and the maximum positive force was $158.4 \mathrm{kN}$ corresponding the first cycle at this level. The negative maximum drift was $-3.28 \%$ (the corresponding drift level in the loading protocol was $-3.5 \%$ ), and the maximum negative force was $-355 \mathrm{kN}$ corresponding the first cycle at this level. One side of the REDC core plate was fractured when the loading drift increased to $-3.27 \%$ (the corresponding drift level in the loading protocol was the first $-3.5 \%$ drift cycle), and the other side of the REDC core plate was fractured when the loading drift increased to $1.93 \%$ (the corresponding drift level in the loading protocol 
1 was the first $3.5 \%$ drift cycle).

2 In the test 3, as shown in Fig. 7(c), the positive maximum drift sustained in Test 3 was $1.8 \%$

3 (the corresponding drift level in the loading protocol was $2.0 \%$ ), and the corresponding positive

4 maximum forces was $235.3 \mathrm{kN}$. The negative maximum drift was $-1.81 \%$ (the corresponding drift

5 level in the loading protocol was $-2.0 \%$ ), and the corresponding negative maximum forces was -

$6 \quad 279.7 \mathrm{kN}$. One side of the REDC core plate was fractured when the loading drift increased to -

$7 \quad 1.54 \%$ (the corresponding drift level in the loading protocol was the third $-2.0 \%$ drift cycle), and

8 the other side of the REDC core plate was fractured when the loading drift increased to $-0.71 \%$

9 (the corresponding drift level in the loading protocol was the fourth $-2.0 \%$ drift cycle).

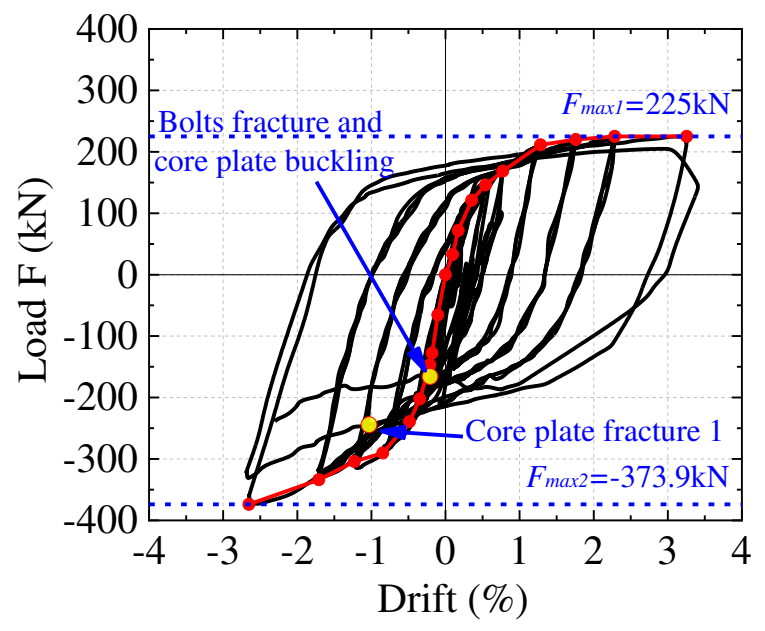

(a) Test 1 (12 mm)

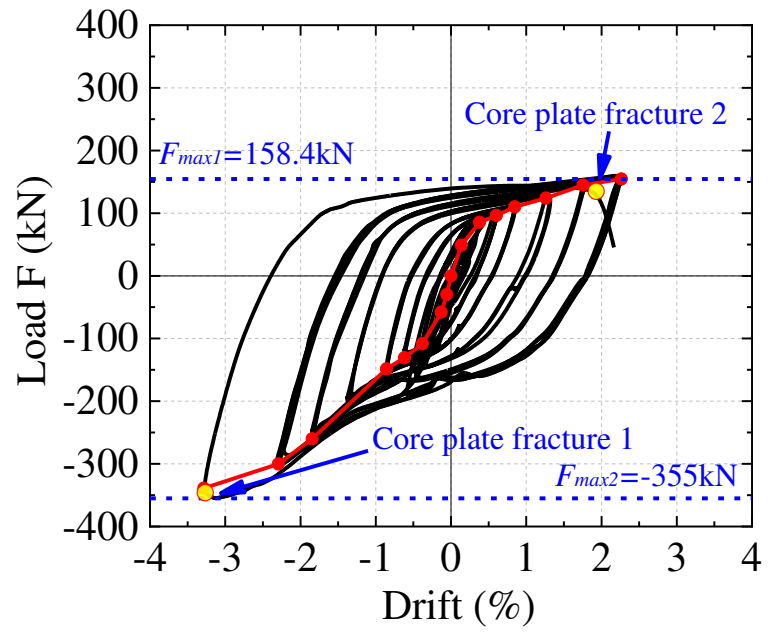

(b) Test $2(10 \mathrm{~mm})$ 


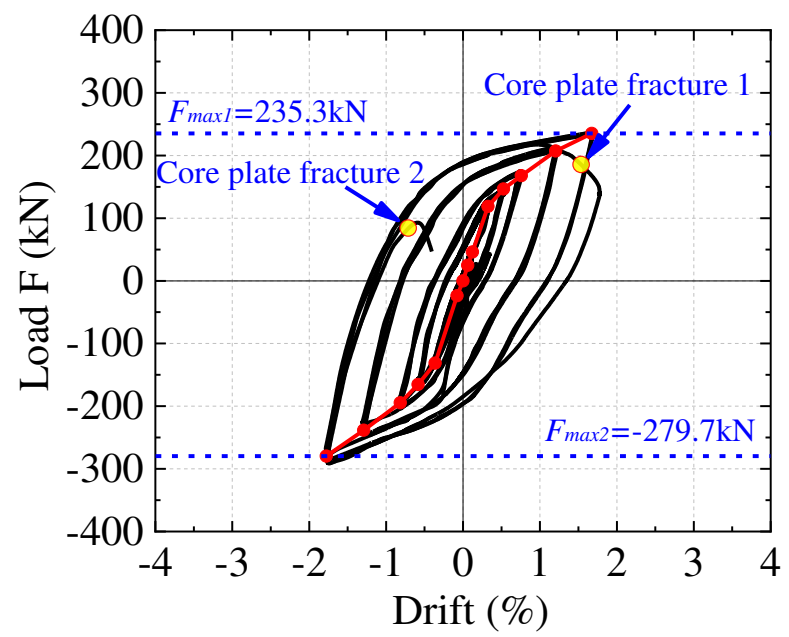

(c) Test $3(14 \mathrm{~mm})$

Fig. 7 Load versus drift response for the SYPC connection: (a) Test 1; (b) Test 2; (c) Test 3

\section{$1 \quad$ 4.2.Cracking pattern}

The cracking patterns of the front and back elevation in Tests 1 are shown in Figs. 8(a) and (b), respectively. Compared with Test 1 , there were almost no new cracks in Test 2 , and only a few of the original cracks continued to expand. It is attributed to the thickness of the core plate in Test 2 is smaller than that in Test 1 , so that the loading capacity of Test 2 is smaller than that in Test 1. In view of this, the cracking pattern in Test 2 is not presented. The cracking patterns of the front and back elevation in Tests 3 are shown in Figs. 8(c) and (d), respectively.

As shown in Fig. 8, the cracks were mainly concentrated in the top-hinge of the beam, including the shear cracks and the flexural cracks. The diagonal cracks were divided into two types: those under a positive drift (A) and those under a negative drift (B). As shown in Fig. 8, these two

11 types of crack originated near the edge of the vertical slots and extended to the top reinforcements of the concrete beam at an angle of approximately $45^{\circ}$. 


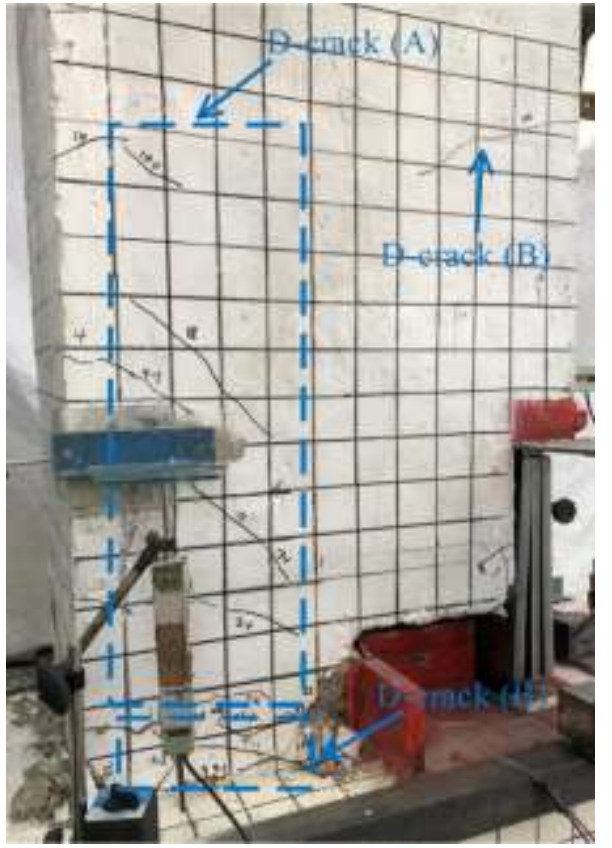

(a) Test 1 (front elevation)

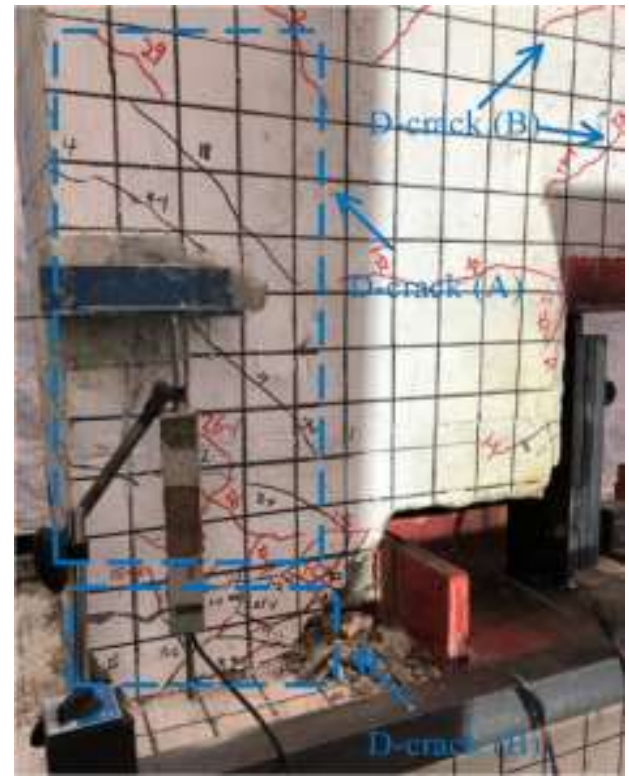

(c) Test 3 (front elevation)

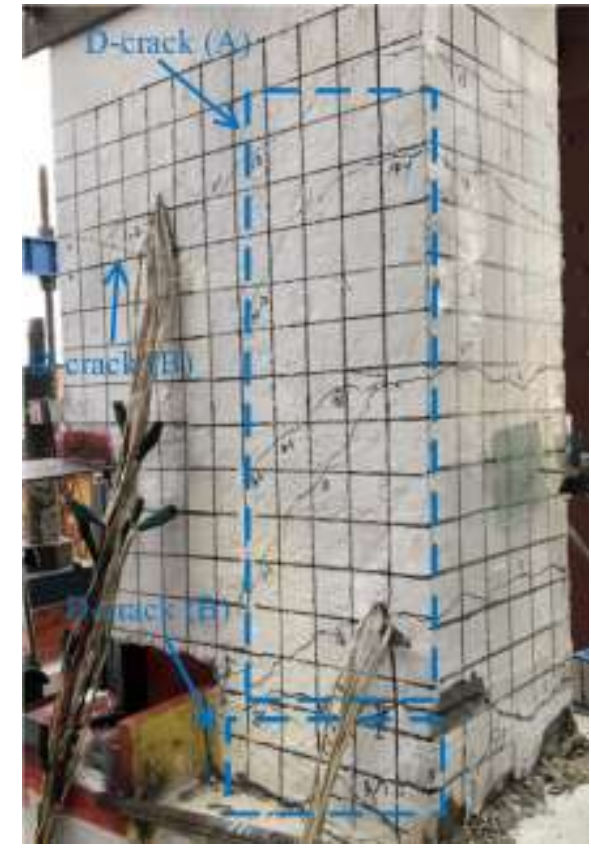

(b) Test 1 (back elevation)

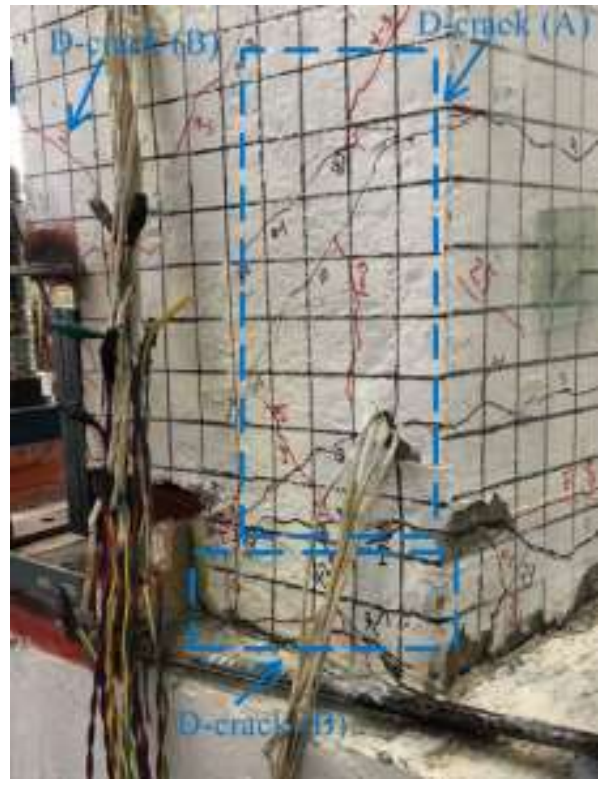

(d) Test 3 (back elevation)

Fig. 8 Cracking patterns at the ultimate condition

1 In Test 1, the first crack occurred in the beam near the beam-column interface at a drift of $0.1 \%$.

2 With an increase in the loading drift (to $0.2 \%, 0.5 \%$, and $1.0 \%$ ), flexural cracks under positive

3 loading developed in the upper part of the beam away from the beam-column interface.

4 Additionally, flexural cracks under negative loading developed in the lower part of the beam. Some 
1 of the flexural cracks developed quickly at the top of the beam and connected with the cracks at

2 the back of the beam, forming through-cracks, which were the main cracks of the connections.

3 When the loading drift increased to $1.0 \%$ and $1.5 \%$, shear cracks developed in the upper part of

4 the beam, which gradually developed from the upper part of the hidden corbel extending into the

5 plastic hinge zone. When the loading drift reached to $-1.5 \%$, the interface of the hidden corbel and

6 the beam was opened. When the drift increased further (to $2.0 \%, 2.5 \%$, and $3.5 \%$ ), the concrete

7 spalled in the upper part of the corbel and the top of the beam near the beam-column interface

8 owing to the compression damage. Because the bending capacity of the connection increased with

9 the increase of the thickness of the REDC in Test 3. New shear cracks and flexural cracks appear in the larger loading drift. But the law of development is similar to Test 1.

\section{$11 \quad$ 4.3.Failure mode}

As shown in Fig. 9(a), the fracture position of the REDC core plate in Test 1 was in the yielding segment of the core plate, where one crack was formed. The REDC core plate were fractured because of the large-strain, low-cycle fatigue. Because the unbonding material was not used in Test 1, a necking phenomenon occurred in the REDC core plate, and several scratches were clearly observed on the REDC core plate, which were caused by the friction between the core plate and the restraint plate. The ordinary bolts (4.8-grade) connected the restraining plates and the filler plates were fractured, as shown in Fig. 10(a). Two full waves were formed on the yielding segment of the REDC core plate. Owing to the buckling of the REDC core plate, the waves amplitudes of the REDC core plate in Test 1 were slightly large. 


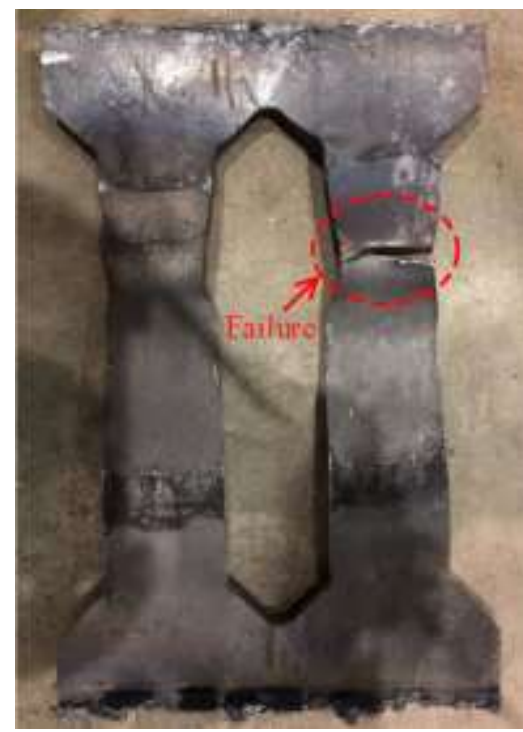

(a) Test 1 (12 mm)

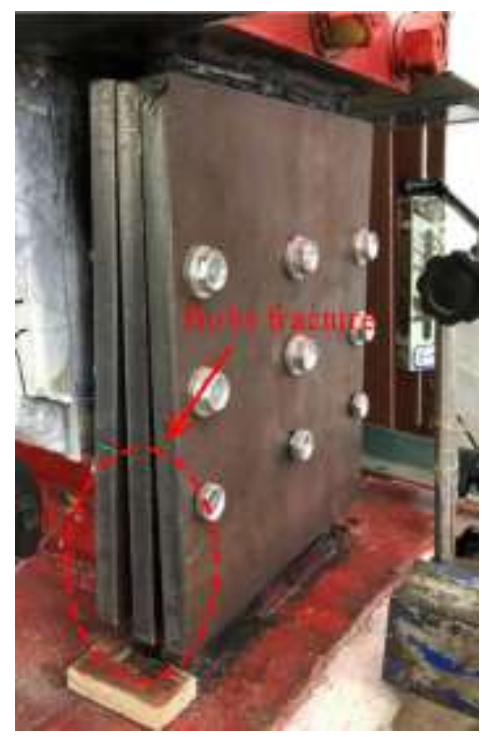

(a) Test $1(12 \mathrm{~mm})$

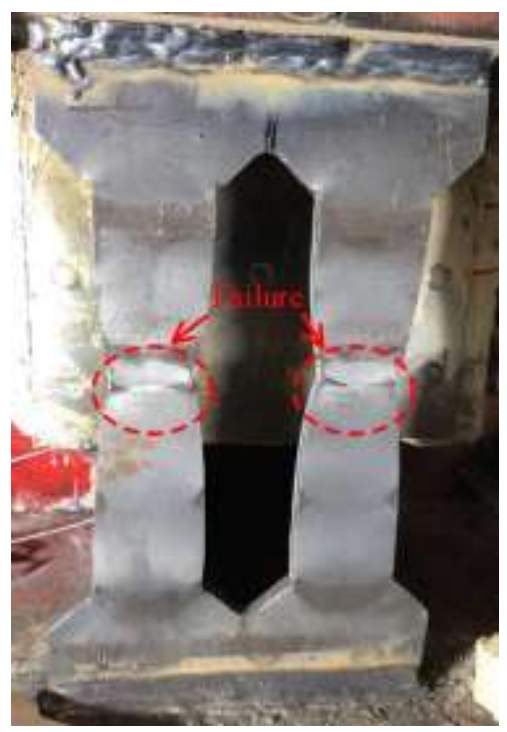

(b) Test $2(10 \mathrm{~mm})$

Fig. 9 Failure pattern of the core plate

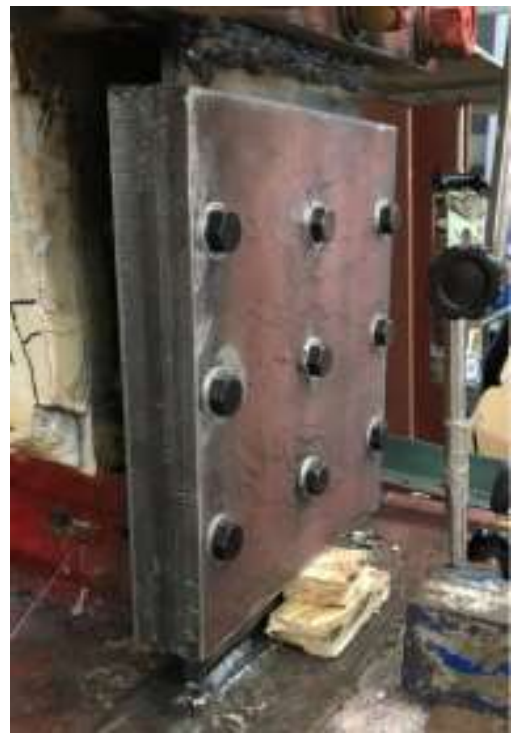

(b) Test $2(10 \mathrm{~mm})$

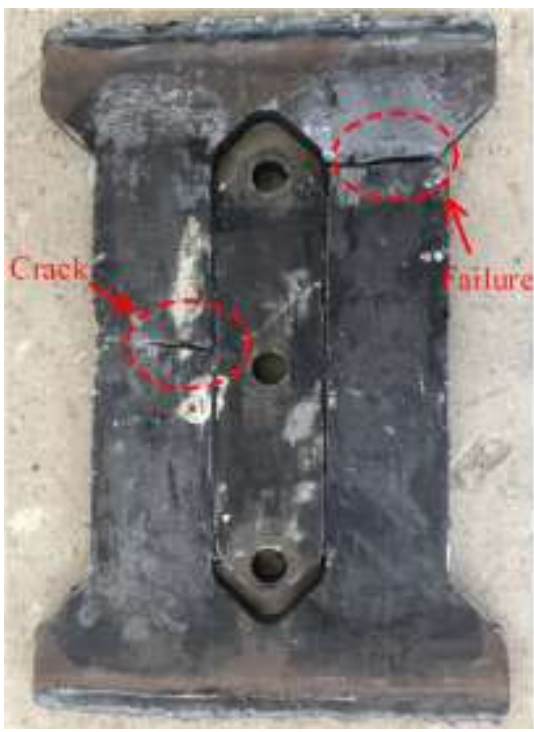

(c) Test 3 (14 mm)

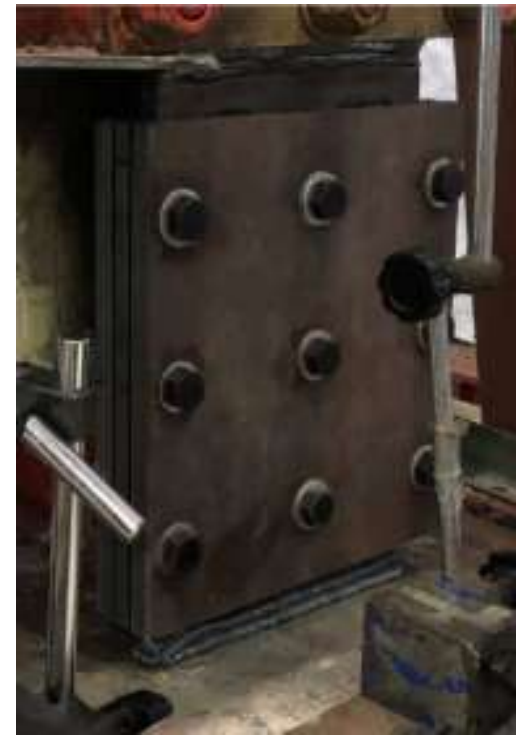

(c) Test $3(14 \mathrm{~mm})$

Fig. 10 Buckling restraint state of the REDC

As shown in Fig. 9(b), because the unbonding material was not used in Test 2, necking

2 occurred in the REDC core plate, and several scratches were clearly observed on the REDC core

3 plate, which were caused by the friction between the core plate and the restraint plate. The fracture

4 position of the REDC core plate in Test 2 was in the yielding segment of the core plate, where two

5 cracks were formed. Because high-strength bolts were used in Test 2, the REDC core plate was 
effectively protected from buckling by the restraint plates, as shown in Fig. 10(b).

As shown in Fig. 9(c), because of the stoppers and the unbonding material was used in Test 3, no scratches were clearly observed in the REDC core plate. The necking phenomenon was not obvious in the REDC core plate. At the same time, the fracture position of the REDC core plate in Test 3 was at the junction of the yielding segment and the transition segment. On the other side, a crack were formed at the middle of the yielding segment of the core plate (near the stopper). Combined with the phenomenon that the drift in Test 3 is relatively smaller than that in Tests 1 and 2 , it can be attributed to the initial defect of the core plate caused by the wire electrical discharge machining (WEDM) technology. In order to realize a 10mm stopper and a $1 \mathrm{~mm}$ gap between the core plate and the filling plate, WEDM technology was easily cause initial defect to the core plate.

This initial defect leaded to early failure of REDC, which limited the ductility of REDC. It can be well proved by the phenomenon of no obvious necking in REDC core plate and its special failure position. Because high-strength bolts (10.9-grade) were used in Test 3, the REDC core plate was effectively protected from buckling by the restraint plates, as shown in Fig. 10(c).

\subsection{Energy dissipation}

The energy-dissipation capability is an important index for evaluating the seismic behavior of structures. The energy dissipation per cycle was used to evaluate the energy dissipation of the novel precast connection. The energy dissipation per cycle of the three tests corresponding to the positive and negative drifts were examined. The energy dissipation of the smaller cycles and the equivalent viscous damping ratio of the smaller cycles and the incomplete final cycles were ignored. The energy dissipation per cycle is plotted in Figs. 11. 
With the gap opening and closing, the energy dissipation per cycle of the novel precast connection exhibited an almost symmetrical "V" shape, as shown in Fig. 10. In the three tests, the energy dissipation per cycle was relatively small when the drift ratio was small $(<0.5 \%)$. However,

4 it increased significantly with the increase of the drift ratio. As indicated by Fig. 11, the energy dissipation per cycle in Test 3 (under both the positive and negative drifts) was greater than that in Test 1 , and the energy dissipation per cycle in Test 1 was greater than that in Test 2 . The results suggest that the energy dissipation per cycle of the novel precast connection increased with the thickness of the REDC core plate. Additionally, the difference in the energy dissipation per cycle dissipation capacity of the novel precast connection in Test 1 consisted of the energy-dissipation capacity of the novel precast connection was only provided by the REDC, because the plastic hinge load level, the energy dissipation values per cycle under positive and negative displacement ratios are approximate, indicating that the energy dissipation capacity of the novel precast connection is good under both positive and negative bending moments. 


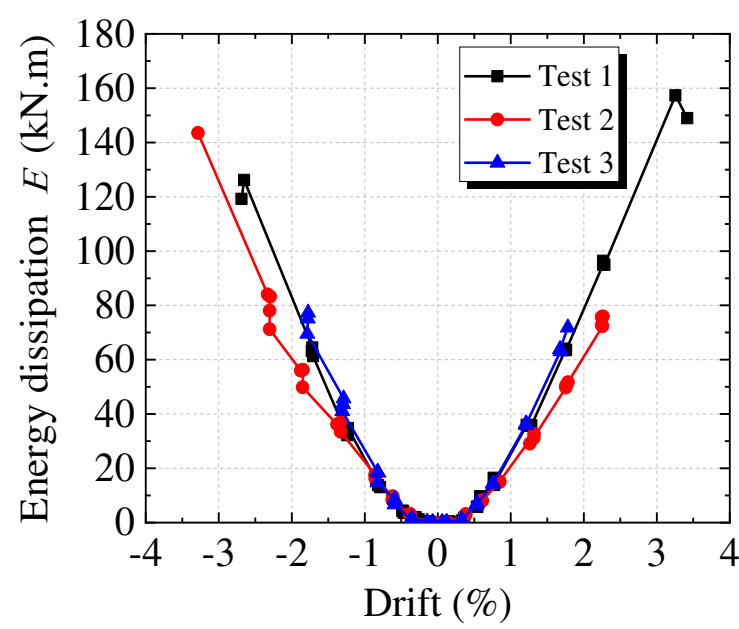

Fig. 11 Energy dissipation per cycle versus drift ratio.

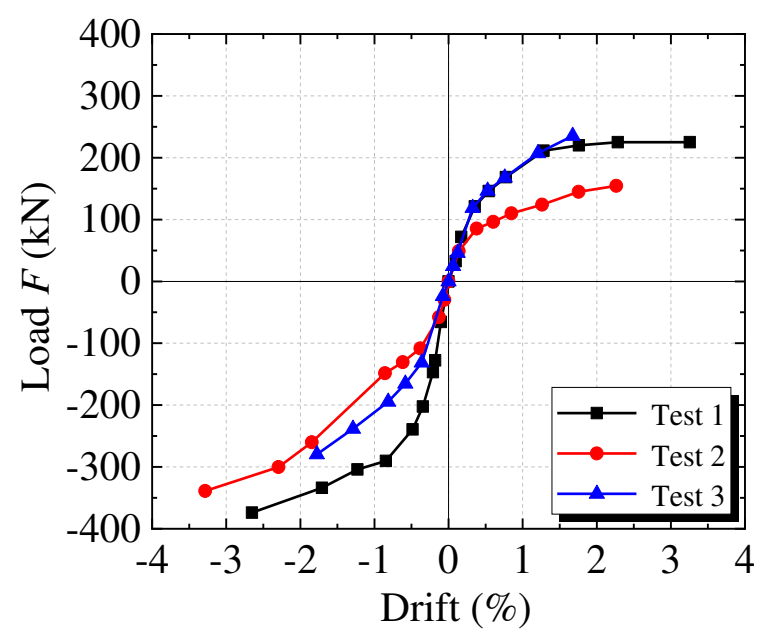

Fig. 12 Skeleton curve

\section{1} 11 directly influenced by the parameters of the REDC.

\subsection{Skeleton curve}

The skeleton curves of the three tests are presented in Fig. 12. As shown in Fig. 12, the negative force was greater than the positive force at the same drift, and the discrepancy was more significant in Tests 1 and 2 than in Test 3. Although the thickness of REDC core plate in Test 1 is less than that in Test 3 . However, the positive force of test 3 is very close to that of test 1 at the same positive drift. This is attributed to the contribution of the concrete compression zone in Test 1. In addition, the positive force in Test 1 was greater than that in Test 2 . The results are attributed to the differences in the thickness of the REDC core plate. At the same time, in Tests 2 and 3, the plastic hinge was completely formed, and the concrete compression zone no longer provided compressive stress. It can be concluded that the load capacity of the novel precast connection is

Additionally, at the same negative drift, the negative force in Test 1 was greater than that in Test 2, and the negative force in Test 2 was greater than that in Test 3. In addition, the difference in the negative force between Tests 1 and 2 was greater than that the difference in the negative 
1 force between Tests 2 and 3. This is most likely because the compressive stress provided by the concrete compression zone made the top reinforcements yield at the initial loading process, then the load-bearing capacity of the connection increased rapidly. As mentioned previously, the plastic hinge was completely formed in Test 1 . The concrete compression zone no longer provided compressive stress in Tests 2 and 3. Another important reason is that the yield stress of the top reinforcements was increased by the "cold drawing" effect after the loading in Test 1, which delayed the yielding of the top reinforcements compared with that in Test 1 . The "cold drawing"

effect means that the yield stress and strain of the reinforcement after a tensile yield is increased compared with the initial state. Additionally, the thickness of the REDC core plate in Test 2 decreased, making the load capacity in Test 2 lower than that in Test 1 . In Test 3, because twice

11 "cold drawing" effect in Tests 1 and 2, the yield stress of the top reinforcements was further increased. Combined with the reason of the fully developed plastic hinge, the load capacity in Test

133 was lower than that in Test 1, although the thickness of the core plate increased.

\subsection{Top longitudinal reinforcement strain profile} specimen, the top longitudinal reinforcement strain in Test 1 was the key to the study. Fig. 13 presents the top longitudinal reinforcement strain profile and the strain history of the top reinforcement at the column face for Test 1. Fig. 13(a) and (a) show the peak strain of the top

19 longitudinal reinforcements corresponding to each peak positive and negative drift, respectively. corresponding drift in the loading protocol is shown in brackets. 


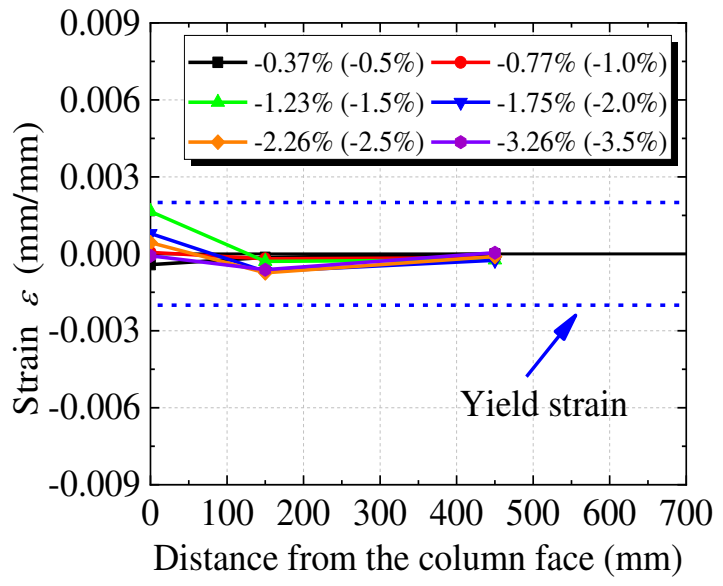

(a) Positive drifts

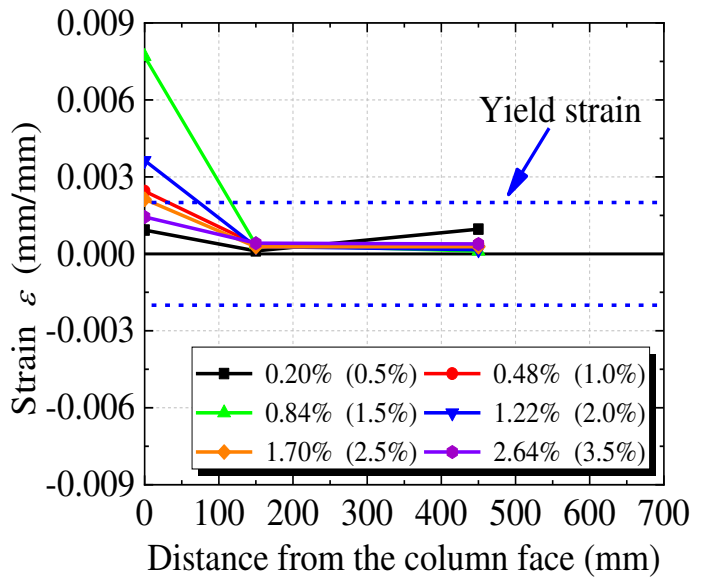

(b) Negative drifts

Fig. 13 Strain profiles of the top reinforcement in Test 1

As shown in Fig. 13, the variation of the top reinforcement strain was the largest at $0 \mathrm{~mm}$ drift (Fig. 13(b)), whereas they were always elastic under the positive drift (Fig. 13(a)). In addition, some tensile strains during gap-opening rotations exhibited when compression would be expected. This is most likely the result of larger residual tensile strains, from previous gap-closing cycles, allowing compressive stresses to occur at positive strains.

Eqs. (5) and (6) are force balance formulas of the connection. After expansion and transformation, Eqs. (7) and (8) can be obtained:

$$
\begin{gathered}
\mathrm{T}_{\mathrm{s}}^{\prime}=\mathrm{C}_{\mathrm{s}}+\mathrm{C}_{\mathrm{c}, \text { bottom }} \\
\mathrm{T}_{\mathrm{s}}=\mathrm{C}_{\mathrm{s}}^{\prime}+\mathrm{C}_{\mathrm{c}, \text { top }} \\
\mathrm{A}_{\mathrm{s}}^{\prime} \mathrm{f}_{\mathrm{s}}^{\prime}=\mathrm{A}_{\mathrm{s}} \mathrm{f}_{\mathrm{y}}+\mathrm{a}_{1} \mathrm{f}_{\mathrm{c}}{ }^{\prime} \beta\left(\mathrm{d}_{\mathrm{c}}-\mathrm{c}\right) \mathrm{b} \\
\mathrm{A}_{\mathrm{s}} \mathrm{f}_{\mathrm{y}}=\mathrm{A}_{\mathrm{s}}^{\prime} \mathrm{f}_{\mathrm{s}}^{\prime}+\mathrm{a}_{1} \mathrm{f}_{\mathrm{c}}^{\prime} \beta \mathrm{cb}
\end{gathered}
$$


1 compression zone $\left(\mathrm{C}_{\mathrm{c}}\right.$, bottom $)$ both increased continuously (Eq. (5)). Although the $\mathrm{A}_{\mathrm{s}}^{\prime} \mathrm{f}_{\mathrm{y}}^{\prime} / \mathrm{A}_{\mathrm{s}} \mathrm{f}_{\mathrm{y}}$ is 3.68

2 (>2.5) in the specimen, the compression force provided by the concrete compression zone $\left(\mathrm{C}_{\mathrm{c}}\right.$,

3 bottom) is very larger. Therefore the tensile stress of the top longitudinal reinforcements quickly

4 increased until the top reinforcements yielded. Under the positive drift, the compression $\left(\mathrm{C}_{\mathrm{s}}\right)$

5 provided by the top longitudinal reinforcements and the compression $\left(\mathrm{C}_{\mathrm{c}}\right.$, top $)$ provided by the concrete compression zone worked together to counteract the tension $\left(\mathrm{T}_{\mathrm{s}}\right)$ provided by the REDC, as shown in Eq. (6). Owing to the limited strain-hardening of the REDC core plate and the $\mathrm{A}_{\mathrm{s}} \mathrm{f}^{\prime}{ }_{\mathrm{y}} / \mathrm{A}_{\mathrm{s}} \mathrm{f}_{\mathrm{y}}$ is $3.68(>2.5)$, the top reinforcements remained elastic at all positive drift.

\subsection{Additional bending moment}

The additional bending moment is an important cause of connection damage. The additional bending moment of the connection is also a major obstacle to the formation of the "strong column and weak beam" mechanism, which also brings troubles to the design. Therefore, this novel precast connection research tries to control the bending moment of the connection by reducing the additional bending moment.

The bending moment of the conventional beam-column connection is produced by the combined action of the pressure generated by the concrete compression zone and the top/bottom reinforcements and the tension generated by the bottom/top reinforcements. If the top and bottom reinforcements in beam is the same, the positive and negative bending moments are as shown in Eq. (9). Then the additional bending moment ( $\left.\mathrm{M}_{\text {additional }}\right)$ produced by the combined action of the pressure generated by the concrete compression zone and the tension generated by the top/bottom reinforcements can be expressed by Eq.(10). 


$$
\begin{gathered}
M_{n}^{ \pm}=A_{s} f_{y}\left(d-d^{\prime}\right)+a_{1} f^{\prime} \beta(d-c) b(d-a / 2) \\
M_{\text {additional }}=a_{1} f^{\prime}{ }_{c} \beta(d-c) b(d-a / 2)
\end{gathered}
$$

The novel beam-column connection proposed in this paper attempts to reduce the height of

2 the concrete compression zone through vertical slot, thereby reducing $\mathrm{M}_{\text {additional. }}$ In addition to the

3 vertical slot, increasing $\mathrm{A}^{\prime}{ }_{\mathrm{s}} \mathrm{f}_{\mathrm{y}} / \mathrm{A}_{\mathrm{s}} \mathrm{f}_{\mathrm{y}}$ is used to reduce the strain of the top reinforcements. Through

4 this structural form, the positive and negative bending moment of the novel beam-column

5 connection strive to be governed by the yielding of the REDC. So as to realize the design goal of

6 controllable bending moment of the connection.

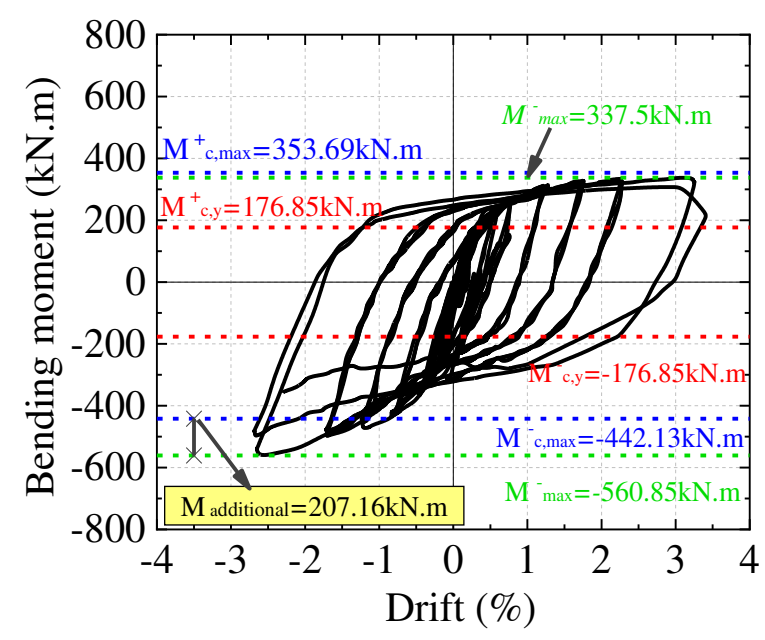

Fig. 14 Schematic diagram of additional bending moment.

Fig.14 is an illustration of the $\mathrm{M}_{\text {additional }}$ discussion with an example of Test $1 . \mathrm{M}_{\mathrm{c}, \mathrm{y}}^{+}$and $\mathrm{M}_{\mathrm{c}}^{-}$,

8 y are the positive and negative yield bending moment calculated by multiplying the yield force of

9 the REDC about a lever arm. They can be called the controllable yield bending moment, as shown

10 in Eqs. (11) and (12). $\mathrm{M}_{\mathrm{c} \text {, max }}^{+}$and $\mathrm{M}_{\mathrm{c} \text {, max }}^{-}$are the overstrength bending moment (considering strain

11 hardening of REDC) of the REDC calculated by multiplying the $\mathrm{M}_{\mathrm{c}, \mathrm{y}}^{+}$and $\mathrm{M}_{\mathrm{c}, \mathrm{y}}^{-}$about a

12 overstrength factor as shown in Eqs. (13) and (14), which can be called the maximum controllable 
1 bending moment. The overstrength coefficient of REDC under tension is conservatively taken as

2 2.0. Considering that the REDC is essentially a BRB, when the BRB is compressed, due to the

3 friction between the core plate and the restraining plate, the compression force of the REDC will

4 be higher than the tension force. Therefore, the overstrength coefficient of REDC under

5 compression is conservatively taken as 2.5 . When $\mathrm{M}_{\text {additional }}$ is zero, the height of the vertical slot

6 in the connection is close to the $d=h-\left(d^{\prime}+1 / 2 d_{\text {reinforcemet }}\right)$, where $h$ represents the height of beam,

7 dreinforcemet represents the diameter of the top longitudinal reinforcement. In this case, it can be

8 approximately considered that under the positive bending moment, the resultant of the concrete

9 compression zone near the center of the reinforcements. Therefore, a/2 in Eqs. (11) and (13) can be approximately replaced by d'.

$$
\begin{gathered}
\mathrm{M}_{\mathrm{c}, \mathrm{y}}^{+}=\mathrm{A}_{\mathrm{s}} \mathrm{f}_{\mathrm{y}}(\mathrm{d}-\mathrm{a} / 2) \\
\mathrm{M}_{\mathrm{c}, \mathrm{y}}^{-}=\mathrm{A}_{\mathrm{s}} \mathrm{f}_{\mathrm{y}}\left(\mathrm{d}-\mathrm{d}^{\prime}\right) \\
\mathrm{M}_{\mathrm{c}, \max }^{+}=\mathrm{A}_{\mathrm{s}} \mathrm{f}_{\mathrm{y}} \times 2 \times(\mathrm{d}-\mathrm{a} / 2) \\
\mathrm{M}_{\mathrm{c}, \max }^{-}=\mathrm{A}_{\mathrm{s}} \mathrm{f}_{\mathrm{y}} \times 2.5 \times\left(\mathrm{d}-\mathrm{d}^{\prime}\right)
\end{gathered}
$$

11 It can be seen from Fig.14 that the maximum bending moment $\mathrm{M}^{+}{ }_{\max }$ is very close to the maximum controllable bending moment $\mathrm{M}_{\mathrm{c} \text {, max }}^{+}$of the novel beam-column connection. This

13 shows that the positive bending moment of the novel beam-column connection proposed in this

14 paper is governed by the yielding of the REDC. In other words, the positive bending moment of

15 the novel beam-column connection is controllable. In addition, the negative maximum bending moment $\mathrm{M}_{\text {max }}^{-}$of the novel beam-column connection is larger than the maximum controllable

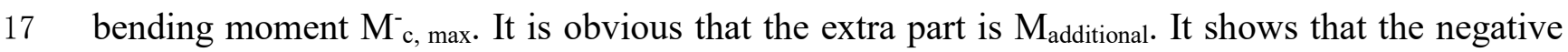


bending moment of the novel beam-column connection is not completely governed by the yielding of the REDC, and extra part is controlled by the compression force generated by the concrete compression zone. This can also be clearly seen from the Eq. (2). However, it is worth mentioning that the negative bending moment of novel beam-column connection is smaller than that of the conventional beam-column connection. The $\mathrm{M}_{\text {additional, novel }}$ of the novel beam-column connection proposed in this paper can be expressed by Eq. (15):

$$
M_{\text {additional, novel }}=a_{1} f^{\prime} \beta\left(d_{c}-c\right) b\left(d_{c}-a / 2\right)
$$

Compared with Eq. (10), because the height of the vertical slot is the half of the beam height. Therefore, the $\mathrm{M}_{\text {additional, novel }}$ of the novel beam-column connection is significantly smaller than $\mathrm{M}_{\text {additional }}$ of the conventional beam-column connection.

\section{Decrease top longitudinal reinforcement strain and additional bending moment under} positive drift

$\mathrm{Au}$ (2010) proposed that for the top reinforcement to remain elastic, $\mathrm{A}^{\prime}{ }_{\mathrm{s}} \mathrm{f}_{\mathrm{y}}{ }_{\mathrm{y}} / \mathrm{A}_{\mathrm{s}} \mathrm{f}_{\mathrm{y}}$ must be $>2.5$. Therefore, the $\mathrm{A}^{\prime}{ }_{\mathrm{s}} \mathrm{f}_{\mathrm{y}} / \mathrm{A}_{\mathrm{s}} \mathrm{f}_{\mathrm{y}}$ of this novel precast connection test specimen was $3.68(>2.5)$. However, during the loading process, the top longitudinal reinforcements still yielded. According to the theory analysis in Section 4.6 and 4.7, a method is proposed to decrease top longitudinal reinforcement strain and additional bending moment under positive drift by increasing the height of the slot (decrease the height of the concrete compression zone). At same time, the method is verified by finite-element simulations.

Numerical analyses were performed using ABAQUS, as shown in Fig. 15. The concrete, steel corbel, and REDC were modelled by eight-node three-dimensional (3D) linear brick elements with 
1 reduced integration $(\mathrm{C} 3 \mathrm{D} 8 \mathrm{R})$, and the reinforcements were modelled by two-node 3D linear truss

2 elements (T3D2). The reinforcements were embedded in the concrete. The surface-to-surface

3 hard contact interaction was applied to the interface between the steel corbel and the precast beam,

4 and the friction coefficient was assumed to be 0.35 . The damage plasticity model for concrete in

5 ABAQUS was utilized to simulate the concrete. A bilinear kinematic hardening model considering

6 the stiffness degradation of steel was used to simulate the steel corbels, reinforcements, and REDC.

7 All the parameters in this model were set according to the material test results (Tables.1-3). All the

8 geometric dimensions of the model were identical to those of the test specimen. The boundary

9 conditions of the connection in the model were identical to those in the test. The depths of the top

10 hinge in the three models were $300 \mathrm{~mm}\left(\mathrm{~d}_{\mathrm{c}} / \mathrm{d}=1 / 2\right), 200 \mathrm{~mm}\left(\mathrm{~d}_{\mathrm{c}} / \mathrm{d}=1 / 3\right)$, and $100 \mathrm{~mm}\left(\mathrm{~d}_{\mathrm{c}} / \mathrm{d}=\right.$

11 1/6). To simplify the model and identify the factors influencing the top longitudinal reinforcement

12 strain, a pushover analysis was performed on each model. It was assumed that the REDC would

13 not fail, i.e., could still function, at a drift ratio of $3 \%$.

14 The Fig. 16 compares the response of the $300 \mathrm{~mm}$ analytical model with the experimental

15 response of the Test 1 . Because the finite element model is a pushover analysis, the finite element

16 simulation is compared with the skeleton curve. It shows that the element is capable of accurately

17 modelling the global force-drift response of the subassembly. 


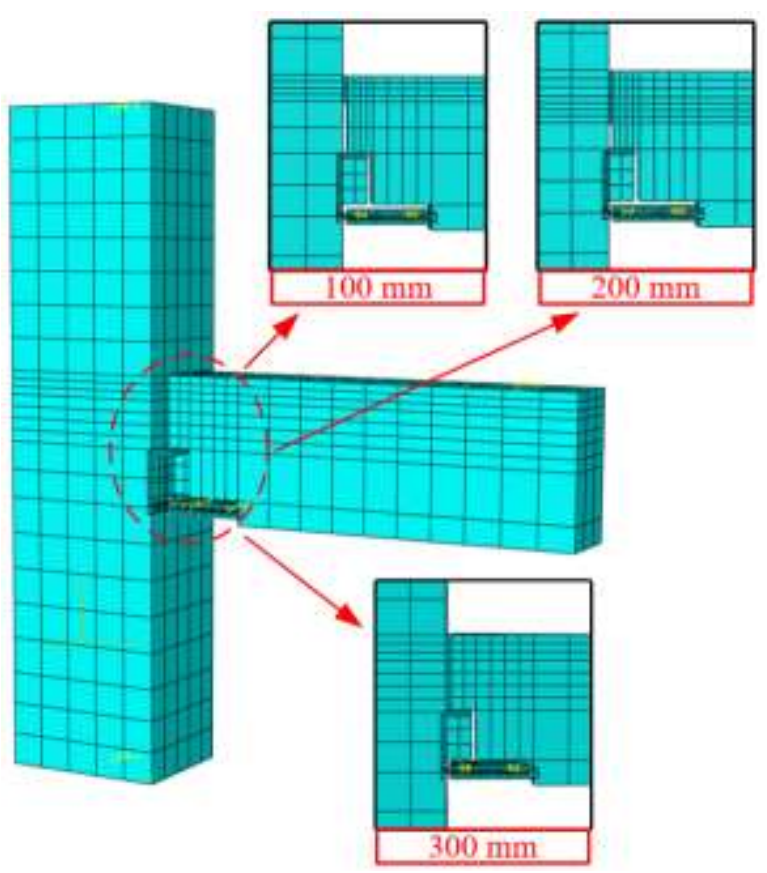

Fig. 15 ABAQUS model for the novel precast connection

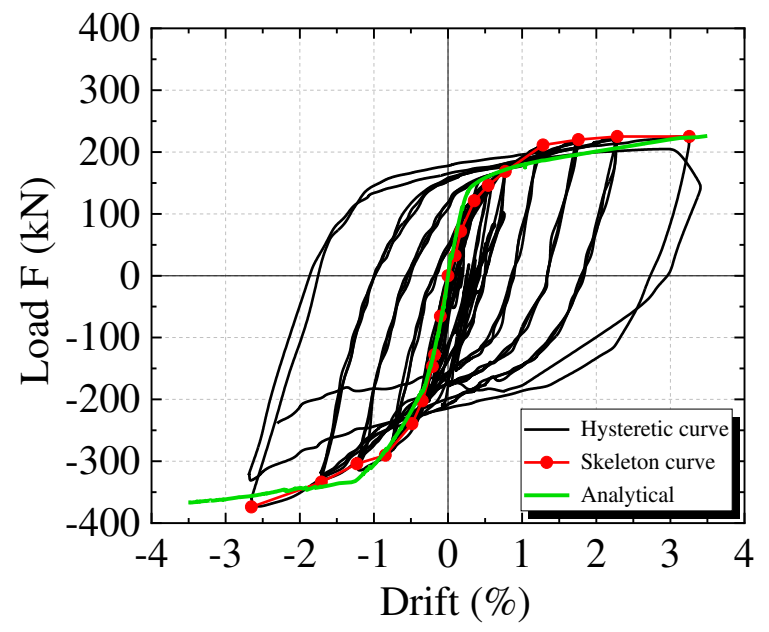

Fig. 16 Comparison of analytical and experimental results
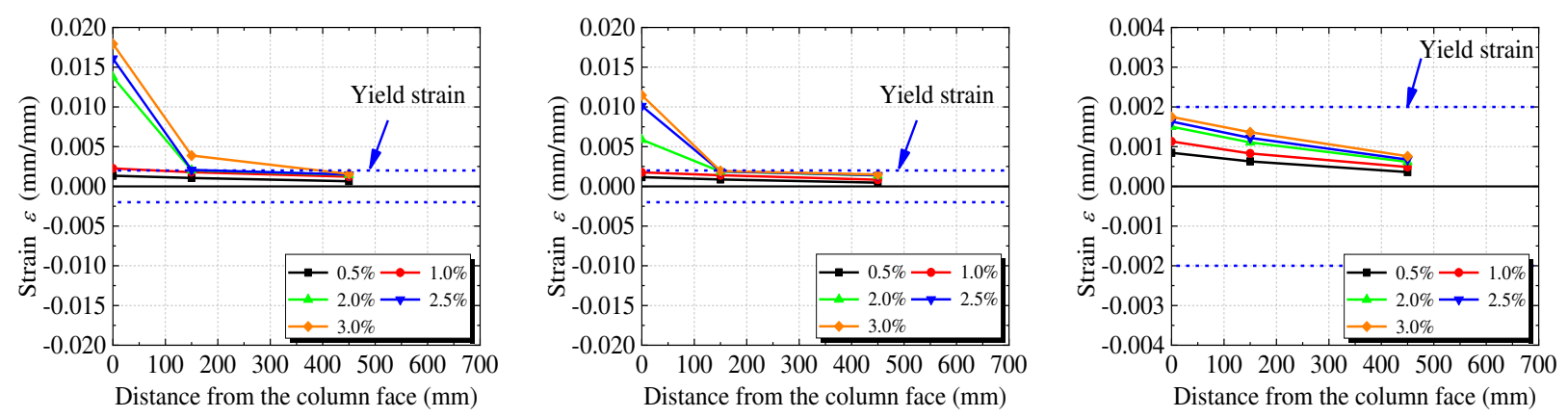

(a) Strain profile in top reinforcement at the

(b) Strain profile in top reinforcement at the negative peak drifts $(200 \mathrm{~mm})$ (c) Strain profile in top reinforcement at the negative peak drifts $(100 \mathrm{~mm})$ negative peak drifts $(300 \mathrm{~mm})$

Fig. 17 The peak strain of the top longitudinal reinforcement corresponding to each positive peak drift in the three models.

The strain of the top longitudinal reinforcement corresponding to negative drifts is the focus

of research. The strain of the top longitudinal reinforcements corresponding to each negative peak

3 drift in the three models is presented in Fig. 17. The strain profiles of the top longitudinal

4 reinforcement in three models were similar to the test result. The variation of the top reinforcement

5 strain was the largest at $0 \mathrm{~mm}$ from the column face. The variations of the top reinforcement strains

6 at 150 and $450 \mathrm{~mm}$ were relatively small. By comparing Fig. 17 (a) - (c), it can be found that the 
1 top reinforcement decreases with the increase of the slot height (the decrease of the top hinge depth). In the model with slot height of 500mm (top hinge depth of 100mm), the strain of top reinforcement corresponding to negative drifts remains elastic. As shown in Figs. 17(a)-(c), with is $100 \mathrm{~mm}$ (vertical slot height is $500 \mathrm{~mm}$ ), the negative maximum bending moment $\mathrm{M}_{\text {max }}^{-}$is close to the negative maximum controllable bending moment $\mathrm{M}_{\mathrm{c} \text {, max }}^{-}$This well confirmed that when the vertical slot height increased to a certain value, the positive and negative bending moment of the novel connection is controllable. In other words, at this time, the positive and negative bending moment of the novel beam-column connection can be governed by the yielding of the REDC.

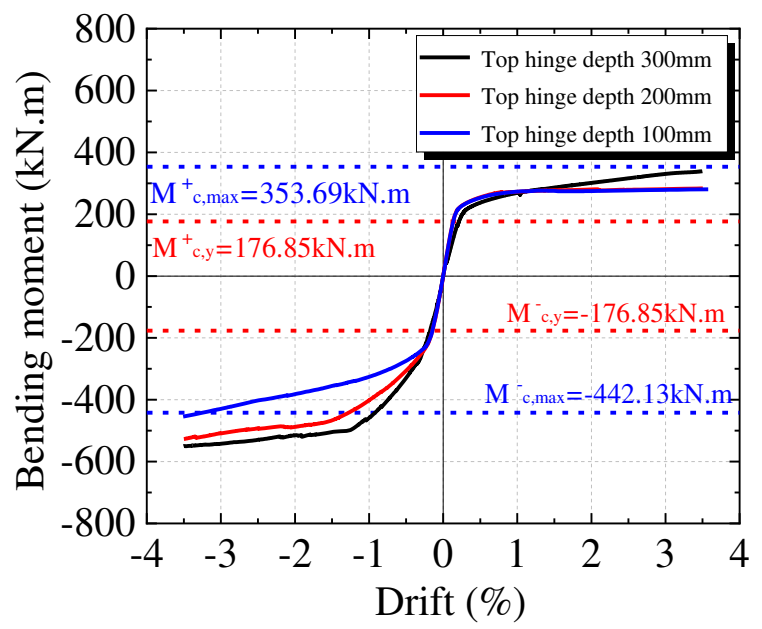

Fig. 18 The comparison of the bending moment

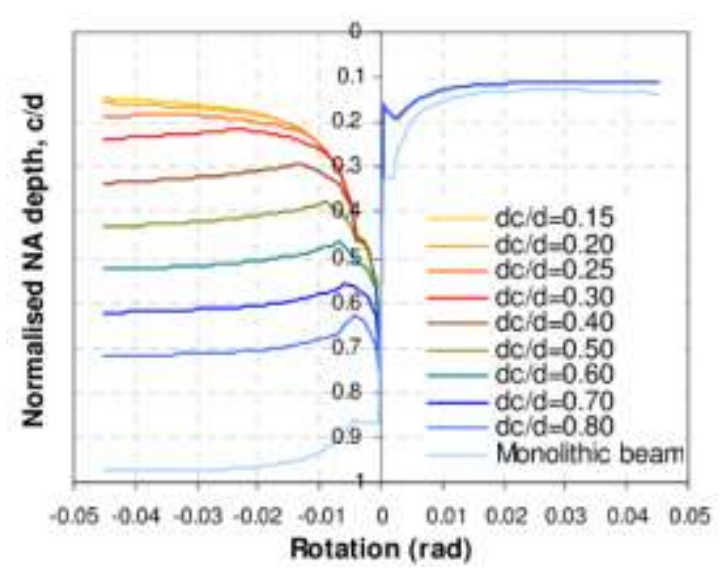

Fig. 19 Parametric study for the concrete top-hinge depth (Au 


\section{Recommended design value of the top hinge depth}

To obtain the recommended design value, further theoretical analysis of the finite-element model is needed. The neutral-axis depth is affected by the height of the vertical slot. When the tophinge depth decreased sufficiently, the neutral axis was infinitely close to the top longitudinal reinforcement of the novel precast connection. According to the force-balance relationship, the position of the neutral axis must be below the top longitudinal reinforcement. As shown in Fig. 19, when the top-hinge depth varied from $0.15 \mathrm{~d}$ to $0.3 \mathrm{~d}$, the position of the neutral axis was close to the top longitudinal reinforcement. Thus, the height of the concrete compression zone $\left(\mathrm{d}_{\mathrm{c}}-\mathrm{c}\right)$ was close to the height of the concrete below the top longitudinal reinforcement in the top hinge $\left(\mathrm{d}_{\mathrm{c}^{-}}\right.$ $d^{\prime}-1 / 2 d_{\text {reinforcemet }}$, where $d_{\text {reinforcemet }}$ represents the diameter of the top longitudinal reinforcement.

11 Therefore, in determining the height of the concrete compression zone and developing a formula

12 for calculating the height of the beam hinge, it is conservative to consider that the neutral-axis

13 position is the position of the lower edge of the top longitudinal reinforcement. When the material

14 properties and geometric dimensions of the connection are known, the safe (maximum) top-hinge 15 depth for ensuring that the top longitudinal reinforcement remains elastic can be calculated as 16 follows:

$$
d_{c}=\left(A_{s}^{\prime} f^{\prime}{ }_{s}-A_{s} f_{s}\right) / \phi a_{1} f^{\prime}{ }_{c} \beta b+c
$$

17 According to this assumption, $\mathrm{c}$ was the distance from the lower edge of the top longitudinal 18 reinforcement to the top surface of the beam, i.e., $\left(d^{\prime}+1 / 2 d_{\text {reinforcemet }}\right)$. When the top-hinge depth is 19 less than or equal to the $d_{c}$ obtained via Eq. (16), the top longitudinal reinforcement does not yield. 
1 Considering the increase of the concrete compression stress due to the constraint influence of stirrups, the amplification coefficient $\phi$ is introduced in Eq. (16). J. B. Mander et al. (1988) proposed the compressive strength of confined concrete can be calculated as follows:

$$
\mathrm{f}_{\mathrm{cc}}^{\prime}=\mathrm{f}_{\mathrm{co}}^{\prime}\left(-1.254+2.254 \sqrt{1+\frac{7.94 \mathrm{f}_{1}^{\prime}}{\mathrm{f}_{\mathrm{co}}^{\prime}}}-2 \frac{\mathrm{f}_{\mathrm{i}}^{\prime}}{\mathrm{f}_{\mathrm{co}}^{\prime}}\right)
$$

4 Therefore, $\phi$ can be calculated as follows:

$$
\phi=-1.254+2.254 \sqrt{1+\frac{7.94 \mathrm{f}_{1}^{\prime}}{\mathrm{f}_{\mathrm{co}}^{\prime}}}-2 \frac{\mathrm{f}_{1}^{\prime}}{\mathrm{f}_{\mathrm{co}}^{\prime}}
$$

5 Where $\mathrm{f}^{\prime}{ }_{\text {co }}$ is the unconfined concrete compressive strength; and $\mathrm{f}^{\prime}{ }_{1}$ is the effective lateral confining stress on the concrete. In order to simplify the calculation, it is approximately specified in this paper that $\phi=1.65$.

According to the material properties and geometric dimensions of the connection of the specimen, the safe top-hinge depth for ensuring that the top longitudinal reinforcement remains elastic is $102.75 \mathrm{~mm}$, smaller than the value of $1 / 4 \mathrm{~d}=150 \mathrm{~mm}$ proposed by $\mathrm{Au}$ (2010). Compared

11 with the constant value of $1 / 4 \mathrm{~d}$, the $\mathrm{d}_{\mathrm{c}}$ obtained via Eq. (16) is influenced by various parameters. Therefore, the top-hinge depth obtained using Eq. (16) is more reasonable, accurate, and safe.

\section{Conclusion}

To realize the controllable bending moment of connections and the principle of "strong column weak beam", a novel precast connection was proposed. According to a theoretical analysis, experimental research, and numerical simulations, the structural form, stress mechanism, and seismic performance of the connection were investigated in detail, and relevant design suggestions were provided. The following conclusions are drawn: 
1 (1) The novel precast connection has the characteristics of a simple structure and easy 2 construction.The REDC is placed at the same horizontal position as the bottom longitudinal 3 reinforcement. The steel corbel is embedded in the column and arranged at the half-height of 4 the beam to effectively transfer the vertical shear force. A vertical slot is installed at the bottom

(4) By reducing the top-hinge depth (increasing the height of the vertical slot), the top longitudinal 
1 reinforcement can be kept in the elastic. At the same time, the designs goal of a "controllable

2 flexural bearing capacity" (reduce the additional bending moment governed by the

3 compression of the concrete compression zone) and the principle of "strong column weak beam"

$4 \quad$ can be realized.

5 (5) A design method and calculation formula for reducing the additional bending moment are

6 proposed. It can provide the design basis for realizing the characteristic of the controllable

7 bending moment. At the same time, this index can be calculated more accurately according to

8 this formula.

\section{Acknowledgments}

The authors would like to acknowledge financial supports from the China National Key

Research and Development Program (2019YFC1509305), the National Natural Science Foundation (51978165).

Amaris A, Pampanin S, Bull D K, Carr A (2008) Experimental investigation on a hybrid jointed

\section{Reference} precast frame with non-tearing floor connections. In: New Zealand Society for Earthquake Engineering Conference, New Zealand.

ASCE/SEI 41-06. (2007) Seismic Rehabilitation of Existing Buildings Published by the American Society of Civil Engineers (ASCE), Prepared by the Structural Engineering Institute of ASCE.

21 Au, E. V. (2010) The mechanics and design of a non-tearing floor connection using slotted 
reinforced concrete beams. Master Dissertation, University of Canterbury, Christchurch, New Zealand.

Bacht T, Chase J G, MacRae G, et al. (2011) HF2V dissipator effects on the performance of a 3 story moment frame. Journal of Constructional Steel Research 67(12): 1843-1849.

Blakeley, Roger W. G., and R. Park. (1971) Seismic Resistance of Prestressed Concrete BeamColumn Assemblies. ACI Structural Journal 68(9): 677-692.

Bull D K. (2003) Understanding the Complexities of Designing Diaphragms in Buildings for Earthquakes. In: Symposium to Celebrate the Lifetime Contributions of Tom Paulay and Bob Park, Christchurch, New Zealand.

C. A. Muir, D. K. Bull, and S. Pampanin. (2013) Seismic testing of the slotted beam detail for reinforced concrete structures. Proceedings of the 2013 Structures Congress 2614-2625.

Chanchi Golondrino, Jose Christian, et al. (2018) Asymmetrical friction connections post-heating behavior. Journal of Constructional Steel Research 149(OCT.): 119-129.

Chanchi Golondrino, Jose Christian, et al. (2019) Asymmetric Friction Connection (AFC) design for seismic energy dissipation. Journal of Constructional Steel Research 157(JUN.): 70-81.

Chang B, Hutchinson T, Wang X, Englekirk R. (2014) Seismic performance of beam-column subassemblies with high-strength steel reinforcement. ACI Structural Journal 111(6): 1329.

Chang B, Hutchinson T C, Wang X, Englekirk R. (2012) Experimental seismic performance of beam-column subassemblies using ductile embeds. Journal of Structural Engineering 139(9): $1555-1566$.

Chi H, Liu J. (2012) Seismic behavior of post-tensioned column base for steel self-centering 
moment resisting frame. Journal of Constructional Steel Research 78: 117-130.

Englekirk, R E. (1995) Development and testing of a ductile connector for assembling precast concrete beams and columns. PCI Journal 40 (2): 36-50.

Englekirk, R E. (1996) An innovative design solution for precast prestressed concrete buildings in high seismic zones. PCI Journal 41(4): 44-53.

Englekirk, R E. (2002) Design-construction of the paramount - a 39-story precast prestressed concrete apartment building. PCI Journal 47 (4): 56-71.

Fan Li., Lv Xilin, Zhao Bin. (2007) Summary of Investigation on Seismic Behavior of Precast Concrete Frame Structures. Structural Engineer 23(4): 90-97. (in Chinese)

Fenwick R C, Davidson B J. (1995) Elongation in ductile seismic-resistant reinforced concrete frames. Recent Developments in Lateral Force Transfer in Buildings, ACI SP-157: 143-170.

Huang X, Eatherton M R, Zhou Z. (2020) Initial stiffness of self-centering systems and application to self-centering-beam moment-frames. Engineering Structures 203: 109890.

Hassoun, M. N., and Al-Manaseer, A. (2020) Structural Concrete: Theory and Design. John wiley \& sons.

Huang X, Zhou Z, Xie Q, et al. (2017) Force distribution analysis of self-centering coupled-beams for moment-resisting-frames without floor elongation. Engineering Structures 147: 328-344 GB50011-2010. (2010) Code for Seismic Design of Buildings (National Standards of the People' s Republic of China). Ministry of Construction of the People's Republic of China. (in Chinese) J.D.R. Byrne, Bull. D K. (2012) Design and testing of reinforced concrete frames incorporating the slotted beam detail. In: New Zealand Society for Earthquake Engineering Conference, 
Kenyon E M. (2008) Predicting the seismic behavior of the Dywidag Ductile Connector (DDC) precast concrete system. Master Dissertation, California Polytechnic State University, San Luis Obispo, United States.

Köken A, Köroğlu M A. (2013) Experimental study on beam-to-column connections of steel frame structures with steel slit dampers. Journal of Performance of Constructed Facilities, 2013, 29(2): 04014066.

Li C, Wu J, Zhang J, et al. (2020) Experimental study on seismic performance of precast concrete frame with replaceable energy-dissipating connectors. Engineering Structures, 231: 111719.

Liu H, Yan Q., Du X. (2017) Seismic performance comparison between precast beam joints and cast-in-place beam joints. Advances in Structural Engineering 20 (9): 1299-1314.

Linjie Huang, Zhen Zhou, Hao Liu, Yi Si. (2020) Experimental Investigation of Hysteretic Performance of Self-Centering Glulam Beam-to-Column Joint with Friction Dampers. Journal of Earthquake and Tsunami DOI: 10.1142/S1793431121500056.

Mander J B, Cheng C-T. (1997) Seismic resistance of bridge piers based on damage avoidance design. Technical Report NCEER-97-0014State University of New York at Buffalo, Buffalo, USA: U.S. National Center for Earthquake Engineering Research (NCEER), Department of Civil and Environmental Engineering.

Mander, J. B., Priestley, M. J., \& Park, R. (1988) Theoretical stress-strain model for confined concrete. Journal of structural engineering, 114(8), 1804-1826.

Mander T J, Rodgers G W, Chase J G, et al. (2009) Damage avoidance design steel beam-column 
moment connection using high-force-to-volume dissipators. Journal of structural engineering 135(11): 1390-1397.

Marriott D. (2009) The development of high-performance post-tensioned rocking systems for the seismic design of structures. Ph.D. Dissertation, University of Canterbury, Christchurch, New Zealand.

Nakaki S D., Englekirk R E, Plaehn J L. (1994) Ductile connectors for a precast concrete frame. PCI Journal 39(5): 46-59.

Ohkubo M, Hamamoto T. (2004) Developing reinforced concrete slotted beam structures to reduce earthquake damage and to enhance seismic structural performance. In: 13th World Conference on Earthquake Engineering. Vancouver, B.C, Canada.

Ohkubo M, Matsuoka T, Yoshioka T, Anderson D L. (2009) Shear transfer mechanism of reinforced concrete beams with a slot at the beam-end. Proceedings of Japan Concrete Institute 21(3), 301-306.

Oh S H, Kim Y J, Ryu H S. (2009) Seismic performance of steel structures with slit dampers. Engineering Structures 31(9): 1997-2008.

Oudah F, El-Hacha R. (2013) Seismic performance of modified single-slotted beam concrete connection. Journal of Earthquake Engineering 21(5):726-751.

Peng B H H, Dhakal R P, Fenwick R C, Carr A J. (2009) Modelling of RC moment resisting frames with precast-prestressed flooring system. In: the 2009 New Zealand Society for Earthquake Engineering, Christchurch, New Zealand, April.

Priestley, M J Nigel., and Macrae G A. (1996) Seismic tests of precast beam-to-column joint 
subassemblages with unbonded tendons. PCI Journal 41(1): 64-81.

Priestley, M J Nigel. (1991) Overview of PRESSS Research Program. PCI Journal (4): 50-57.

Priestley, M J Nigel. (1996) The PRESSS Program-Current Status and Proposed Plans for Phase

III. PCI Journal (2): 22-40.

Rodgers G W. (2009) Next generation structural technologies: Implementing high force-to-volume energy absorbers. Ph.D. Dissertation, University of Canterbury, Christchurch, New Zealand.

Thomphon K J. (1975) Ductility of concrete frames under seismic loading. Ph.D. Dissertation, Department of Civil Engineering, University of Canterbury, New Zealand.

Tong C, Wu J, Hua K, Xie L. (2020) Low-Cycle Fatigue Life Estimation Curve for BucklingRestrained Braces Based on Cumulative Plastic Deformation. Journal of Earthquake Engineering DOI: 10.1080/13632469.2020.1772152.

Tsutomu Usami, Chunlin Wang, Jyunki Funayama. (2011) Low-cycle fatigue tests of a type of buckling-restrained braces. Procedia Engineering 14: 956-964.

Vasconez R M., Naaman A E, Wight J K. (1998) Behavior of HPFRC Connections for Precast Concrete Frames Under Reversed Cyclic Loading. PCI Journal 43(6): 58-71.

Xie Luqi. (2020) Research on the mechanism of replaceable energy dissipation connector and its seismic performance in precast concrete frame joint. Ph.D. Dissertation, Southeast University (China), Nanjing, China. (in Chinese) 


\section{Figures}

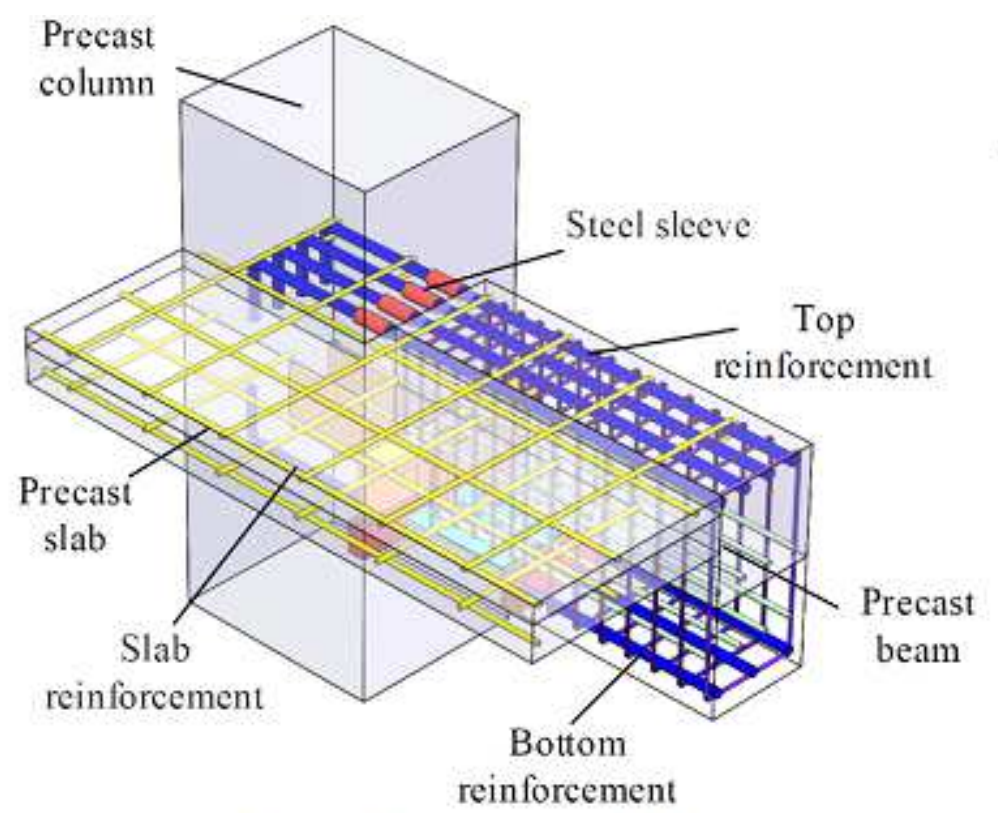

(a) Side elevation (from top)

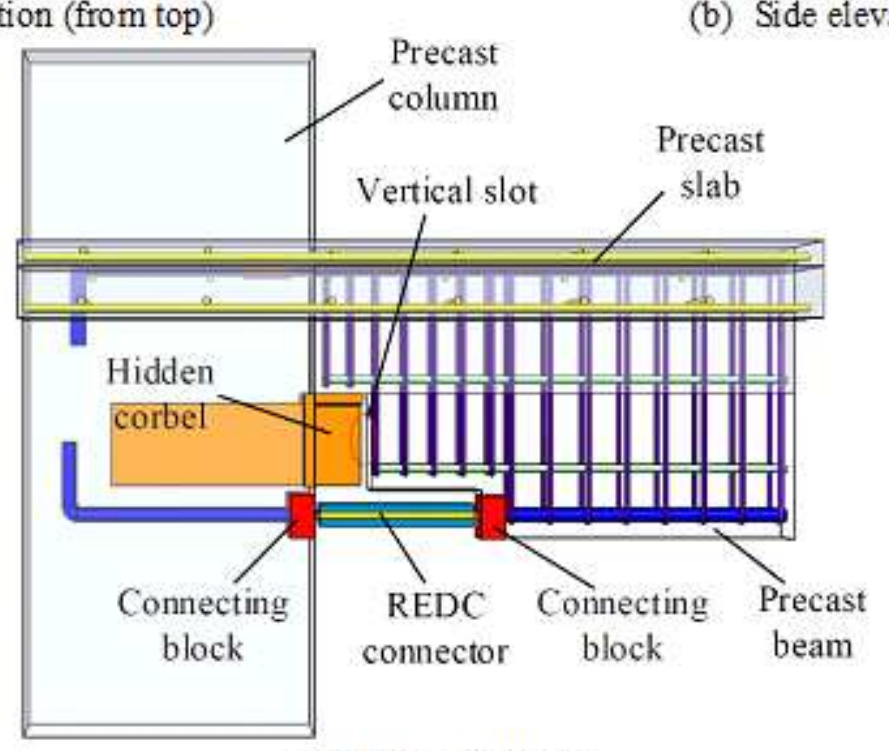

(c) Front elevation

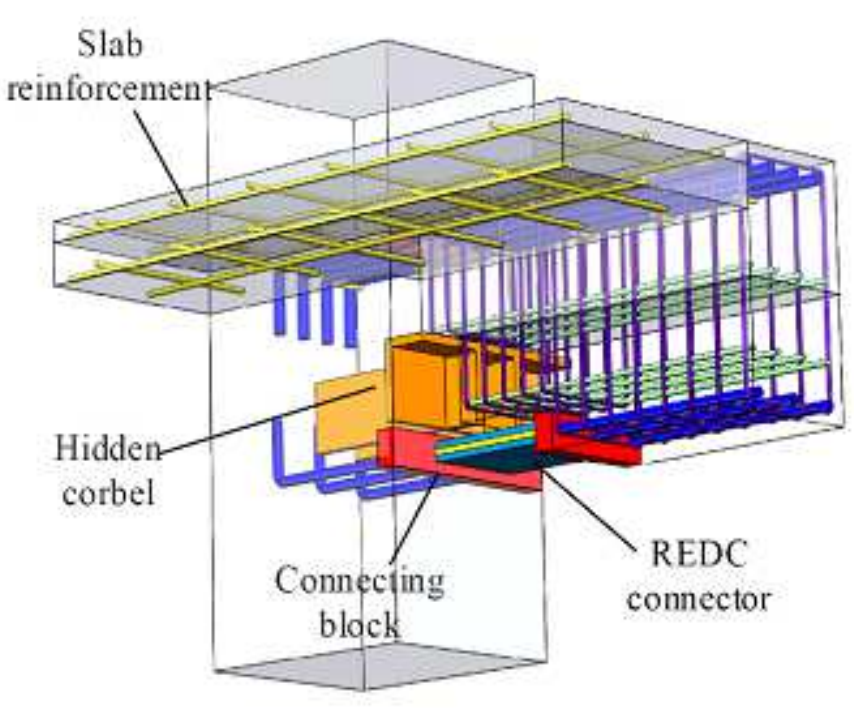

(b) Side elevation (from bottom) 


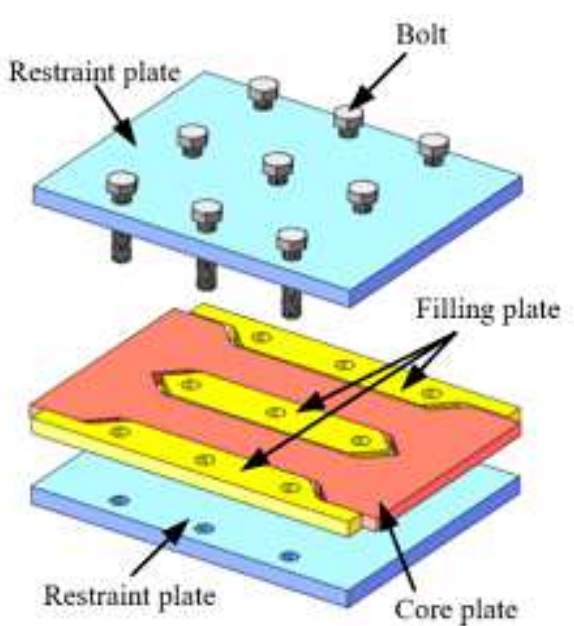

(a) Details of REDC

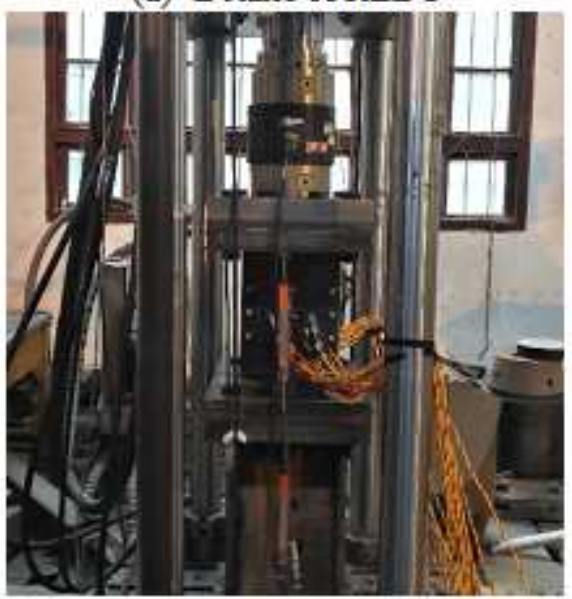

(c) Test of REDC

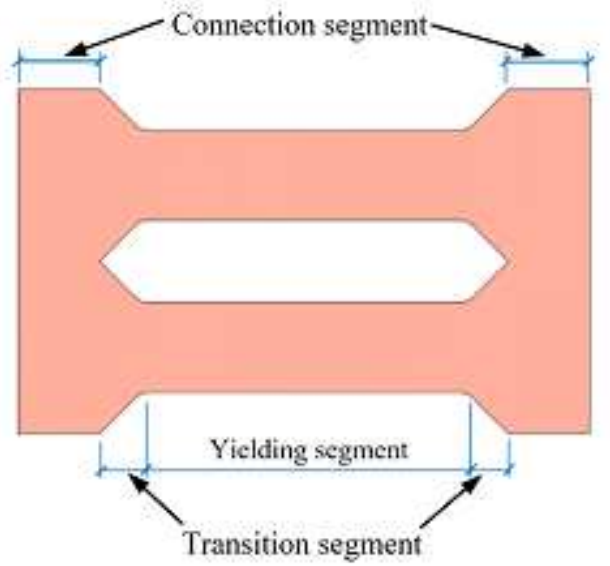

(b) Details of REDC core plate

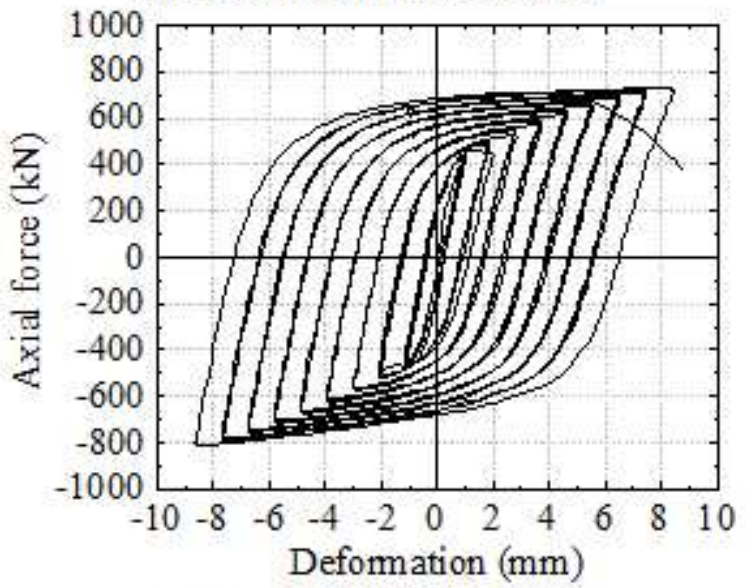

(d) Hysteric curve of REDC

Figure 2

Details and test of REDC

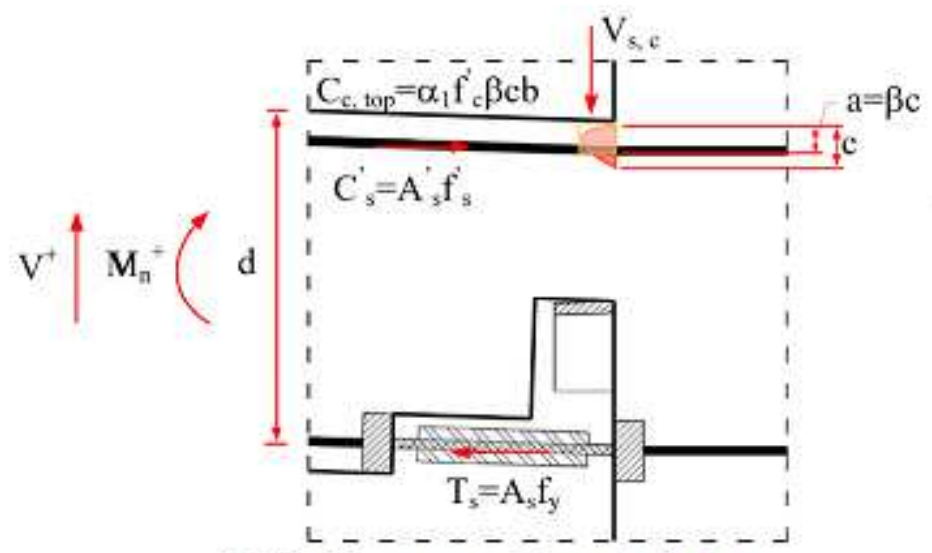

(a) Positive moment (gap closing)

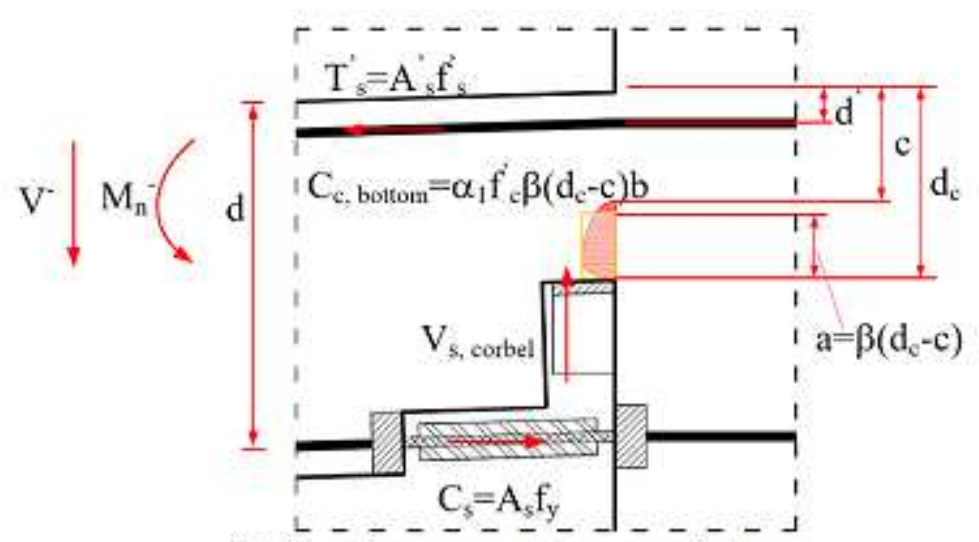

(b) Negative moment (gap opening)

\section{Figure 3}

Force distribution in the connection under positive and negative bending 


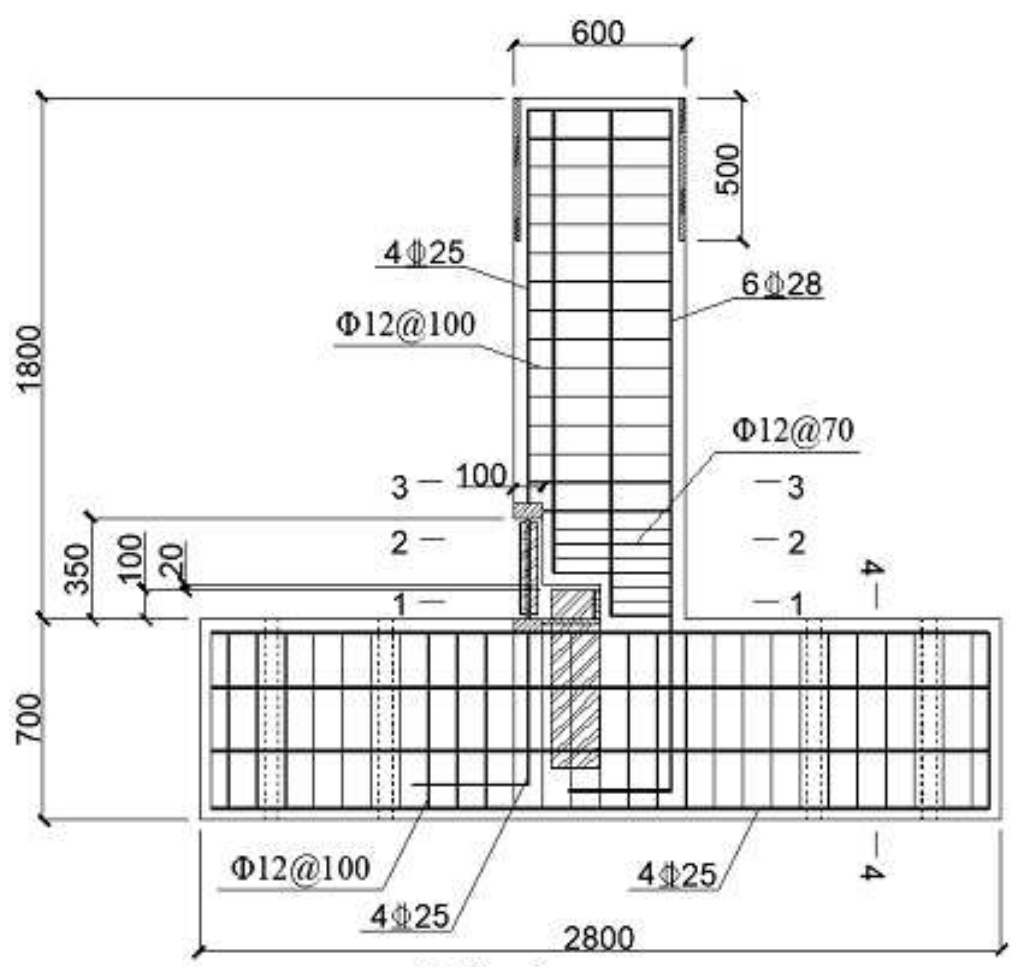

(a) Specimen

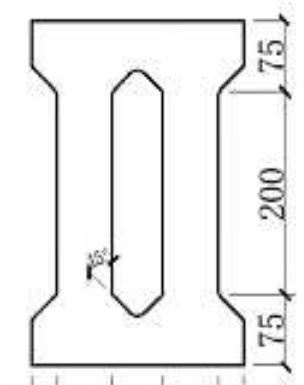

$25,, 55,50,55,25$

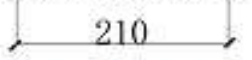

(f) Core plate $(12 / 10 \mathrm{~mm})$

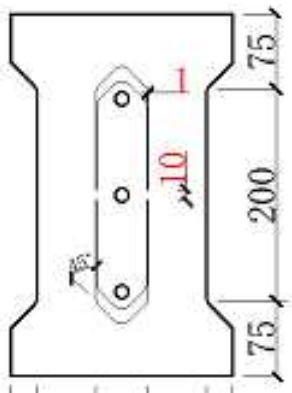

$, 12 / 1025,, 55,50,55,25$ 210

(g) Core plate $(14 \mathrm{~mm})$

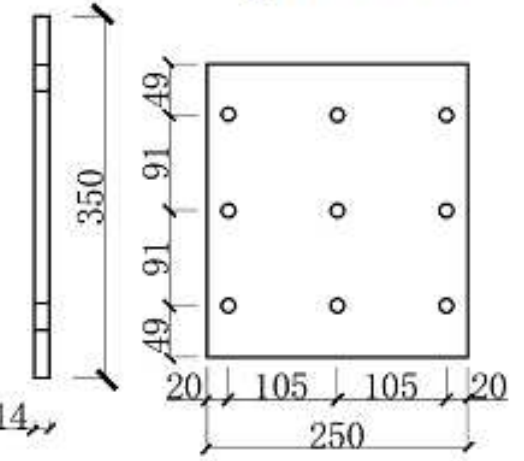

(h) Restraining plate

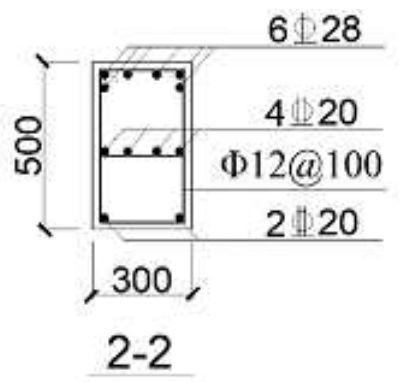

(c) Beam section 2-2

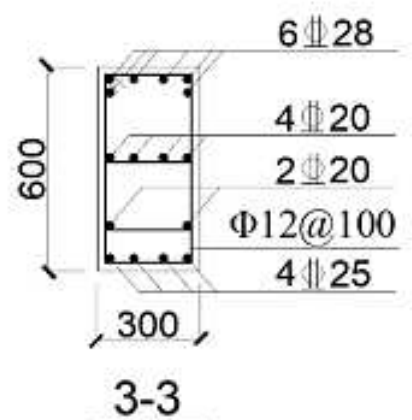

(d) B eam section 3-3

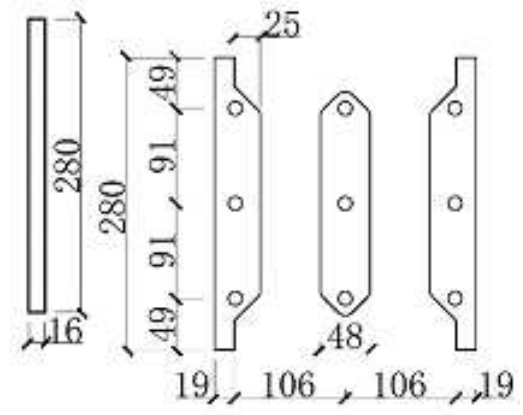

(i) Filler plate

Figure 4

Details of the tested connections (all dimensions have units of $\mathrm{mm}$ )

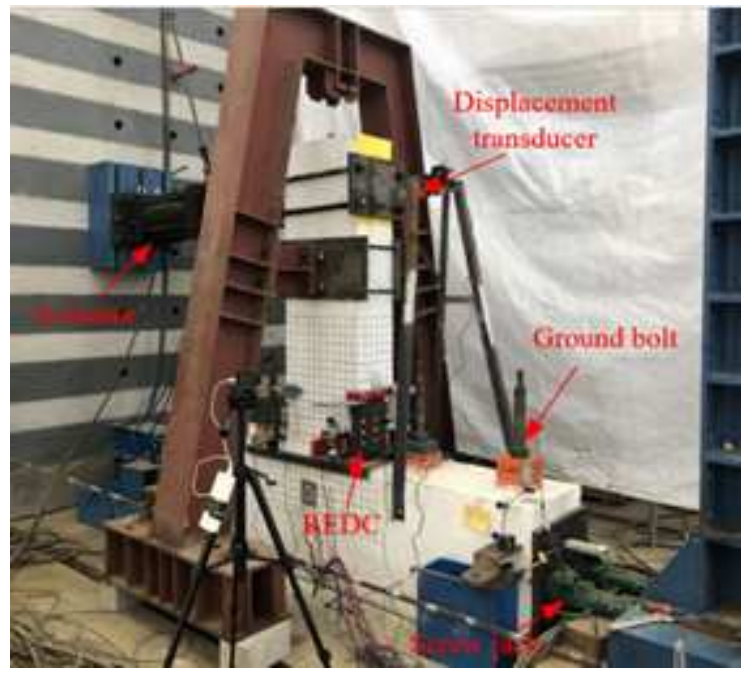


Figure 5

The specimen under test

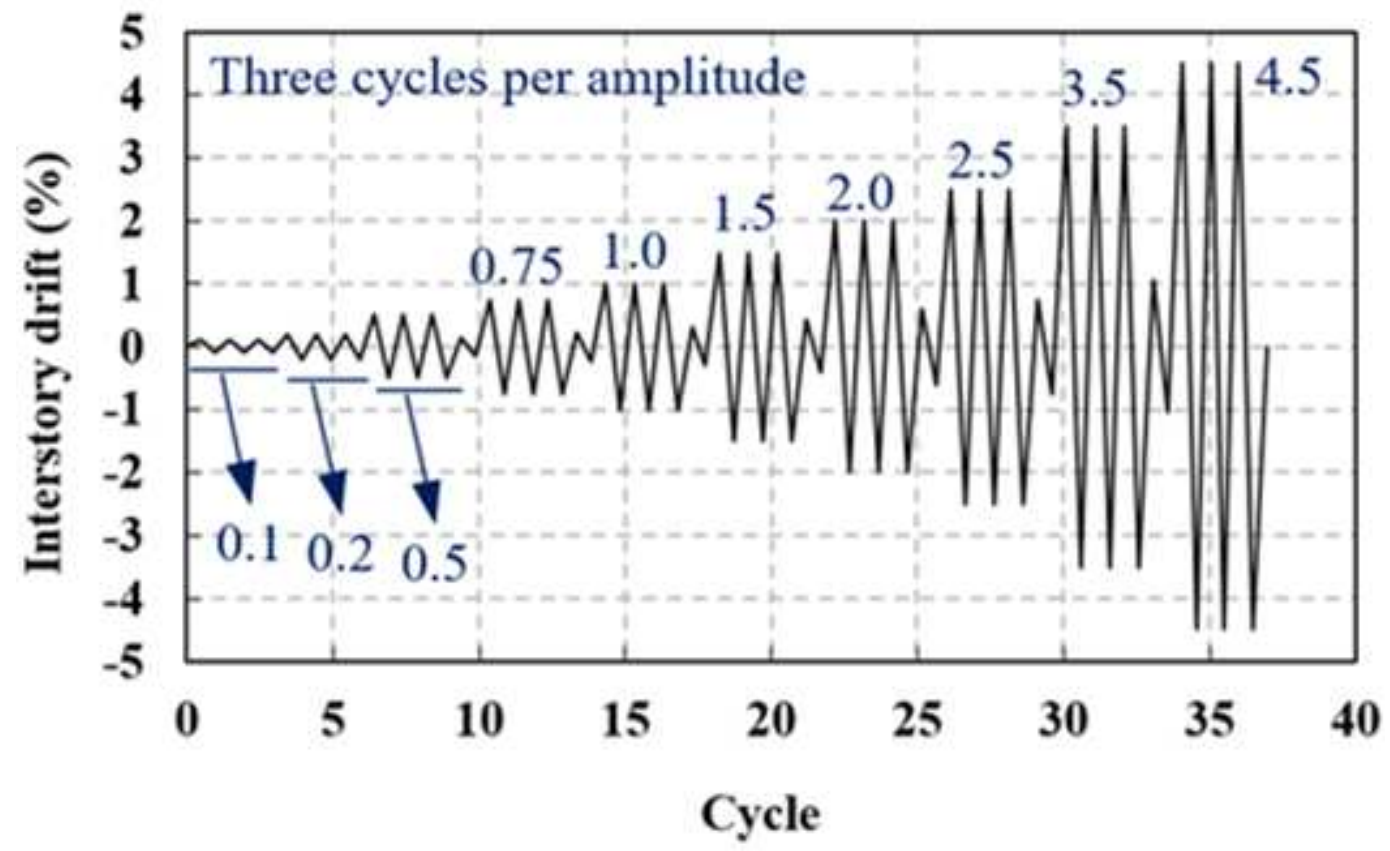

Figure 6

Quasi-static loading 


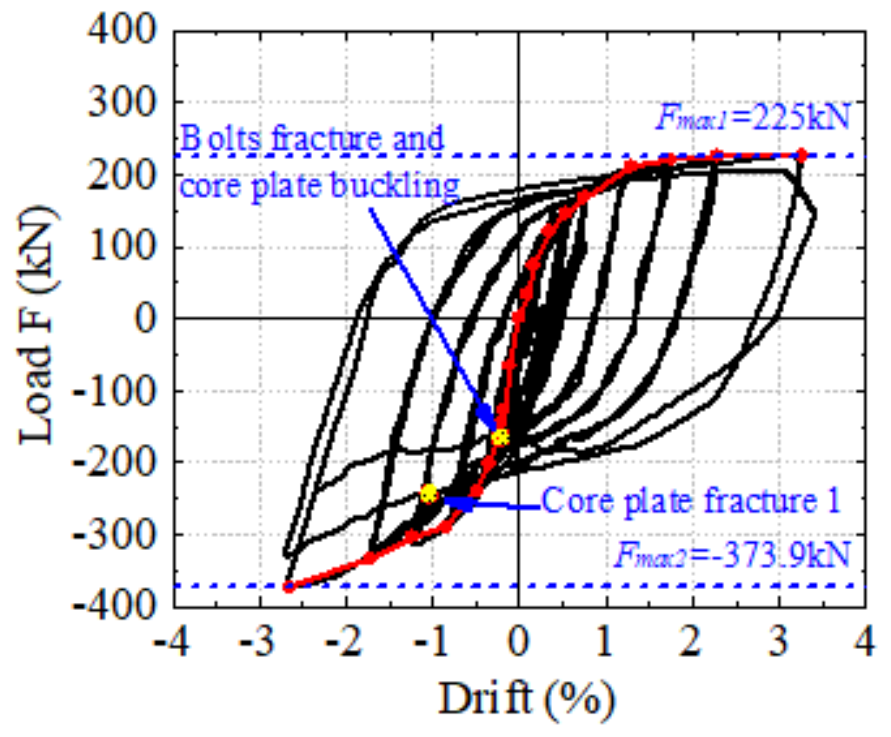

(a) Test $1(12 \mathrm{~mm})$

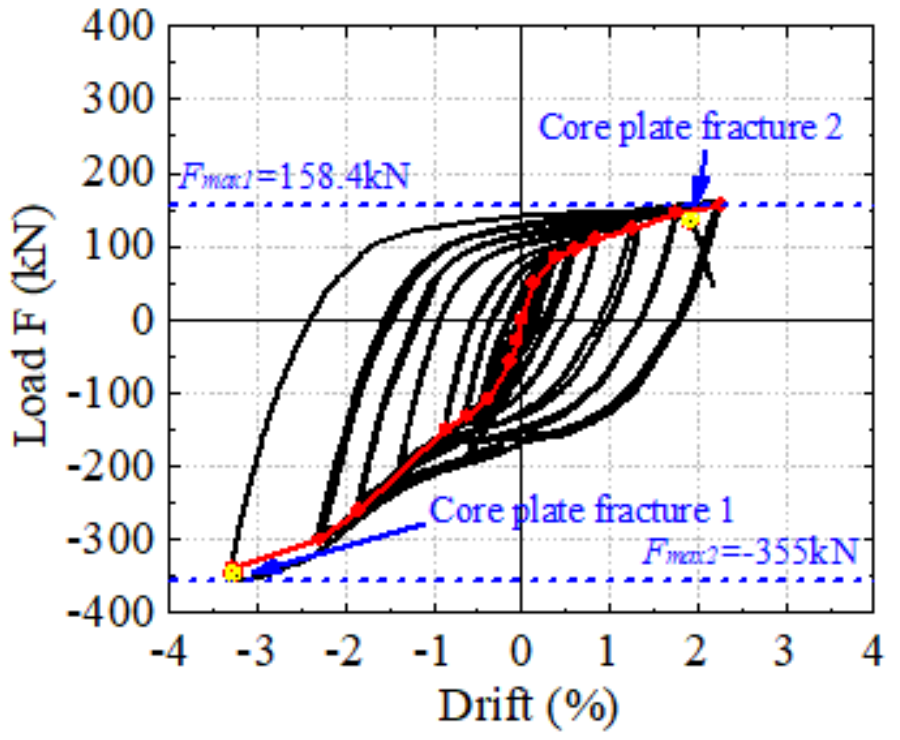

(b) Test $2(10 \mathrm{~mm})$

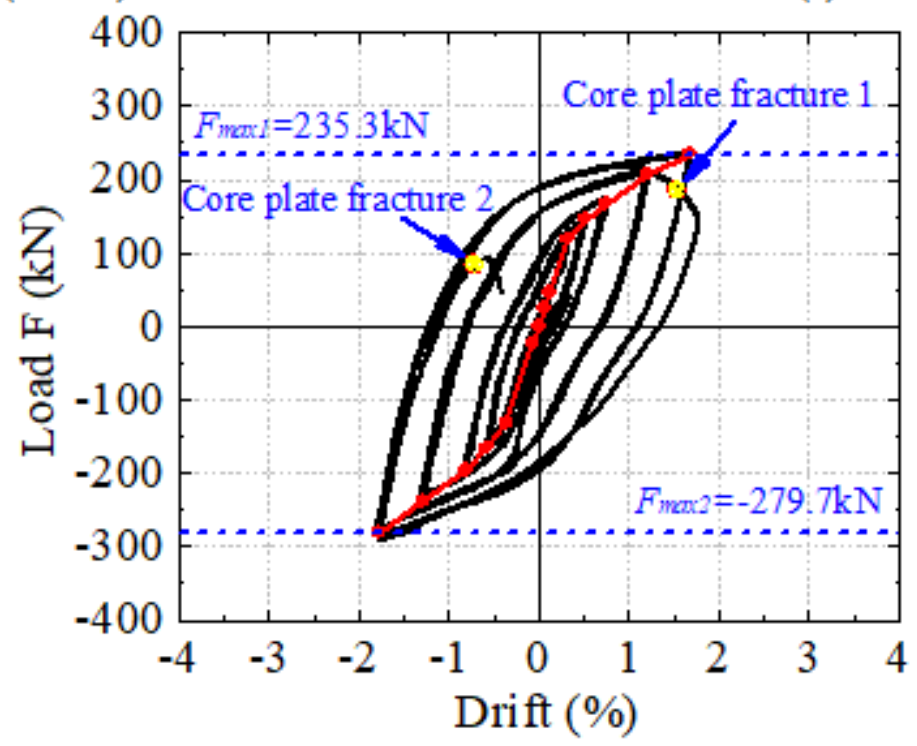

(c) Test $3(14 \mathrm{~mm})$

Figure 7

Load versus drift response for the SYPC connection: (a) Test 1; (b) Test 2; (c) Test 3 


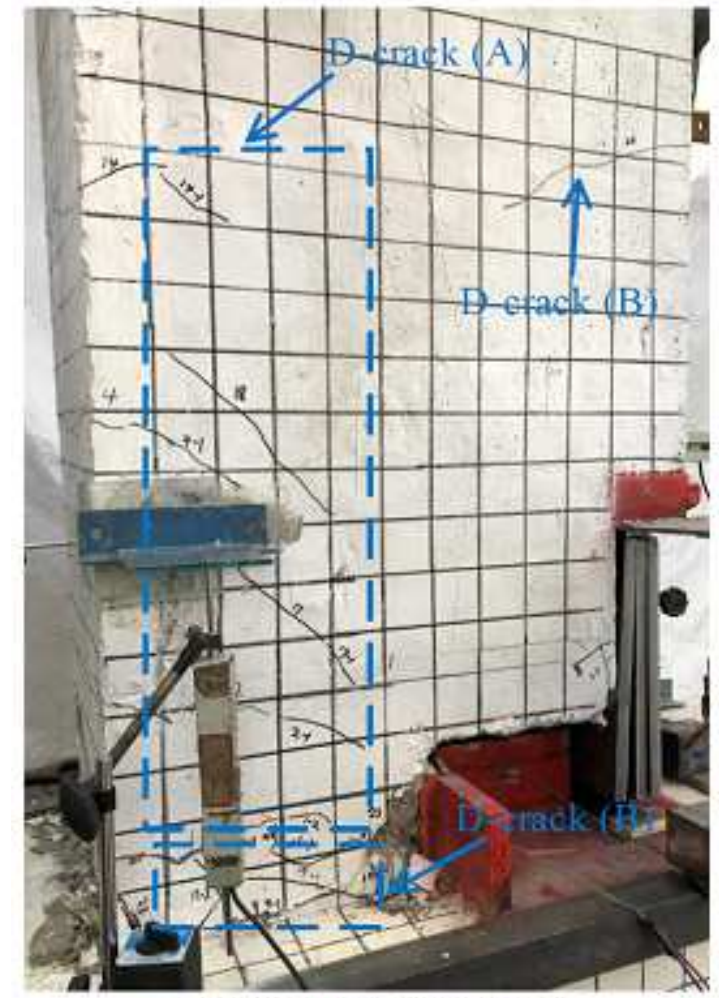

(a) Test 1 (front elevation)

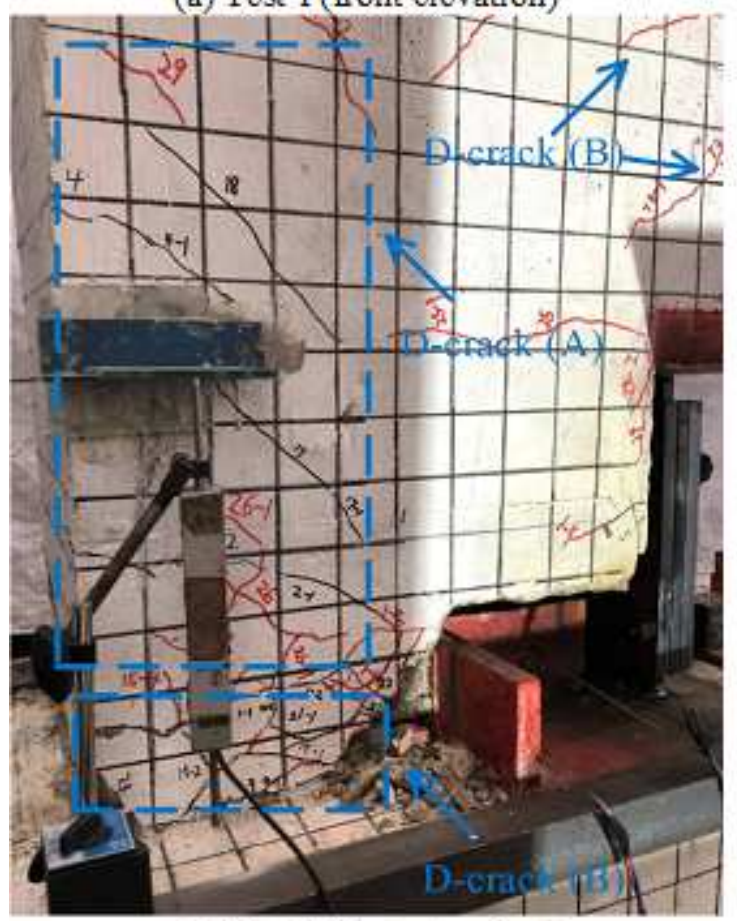

(c) Test 3 (front elevation)

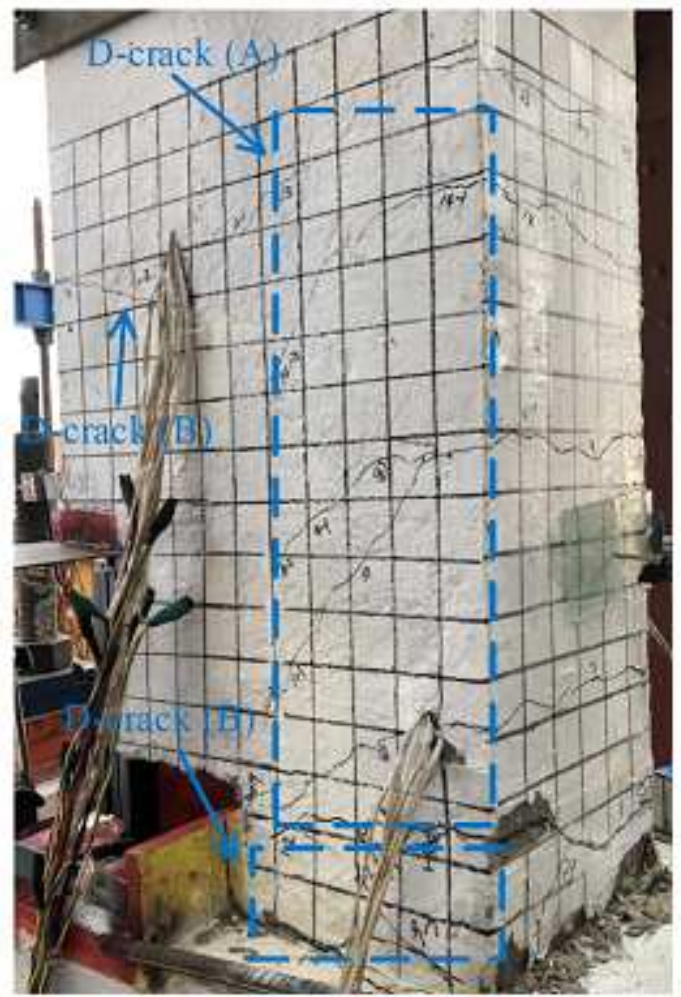

(b) Test 1 (back elevation)

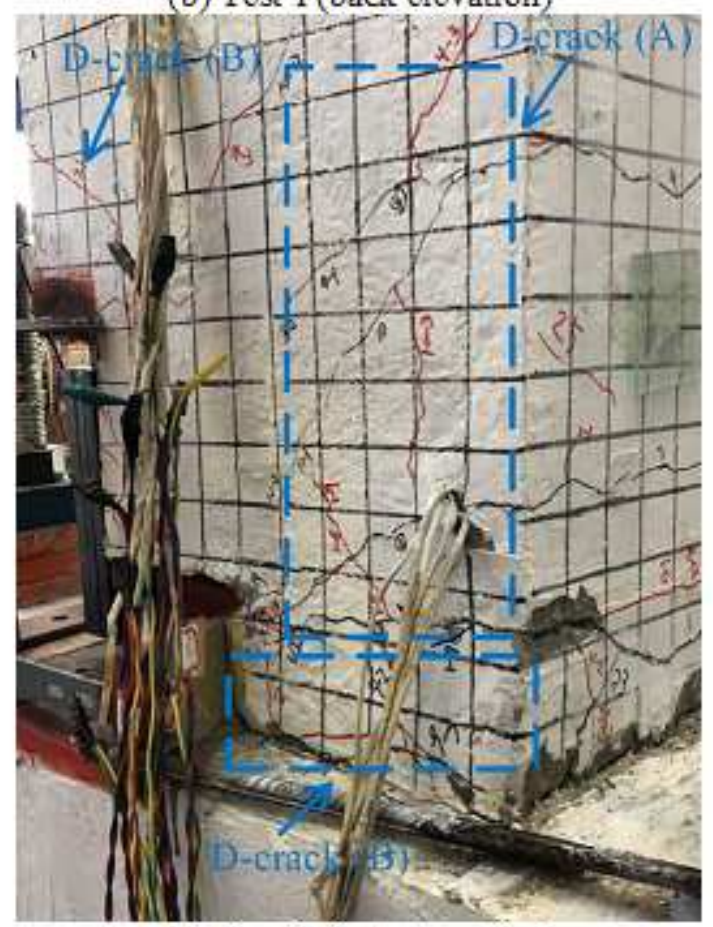

(d) Test 3 (back elevation)

Figure 8

Cracking patterns at the ultimate condition 


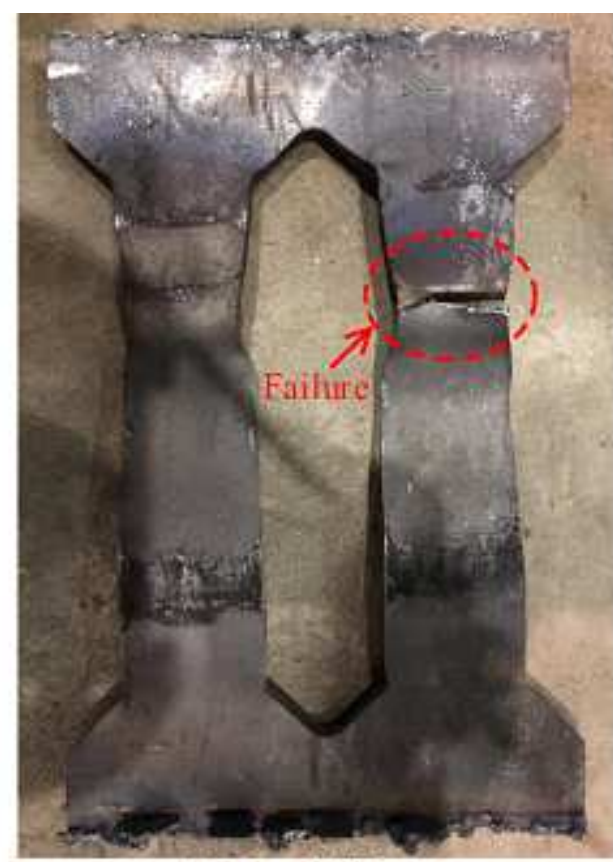

(a) Test $1(12 \mathrm{~mm})$

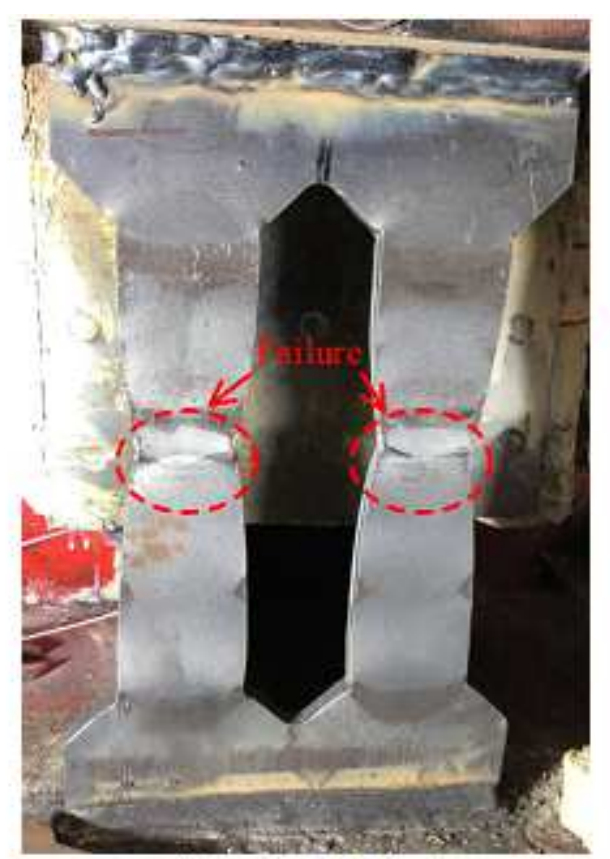

(b) Test $2(10 \mathrm{~mm})$

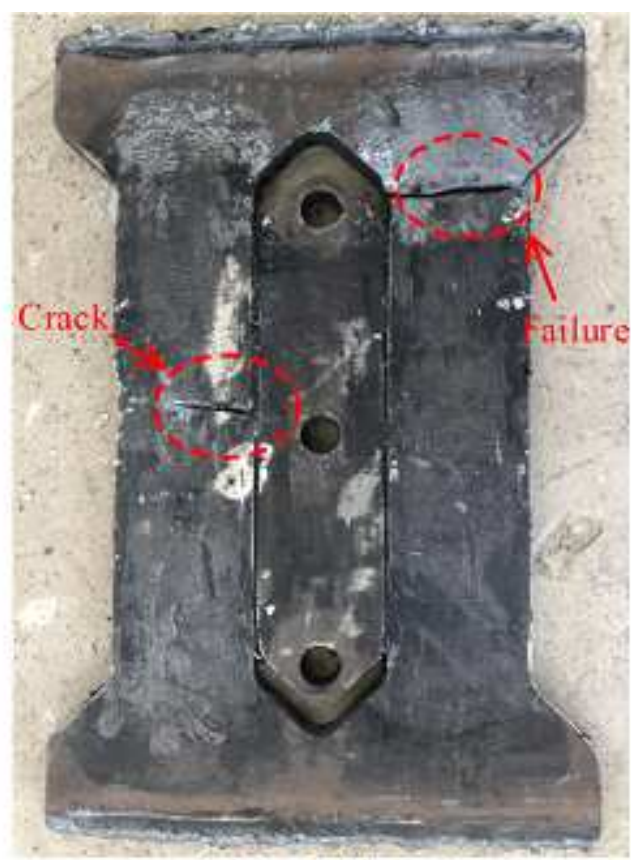

(c) Test $3(14 \mathrm{~mm})$

\section{Figure 9}

Failure pattern of the core plate

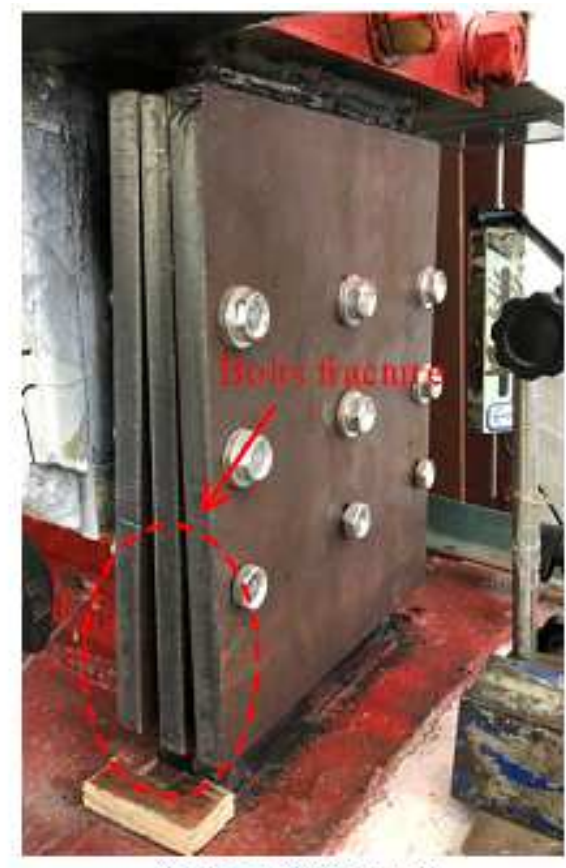

(a) Test 1 (12 mm)

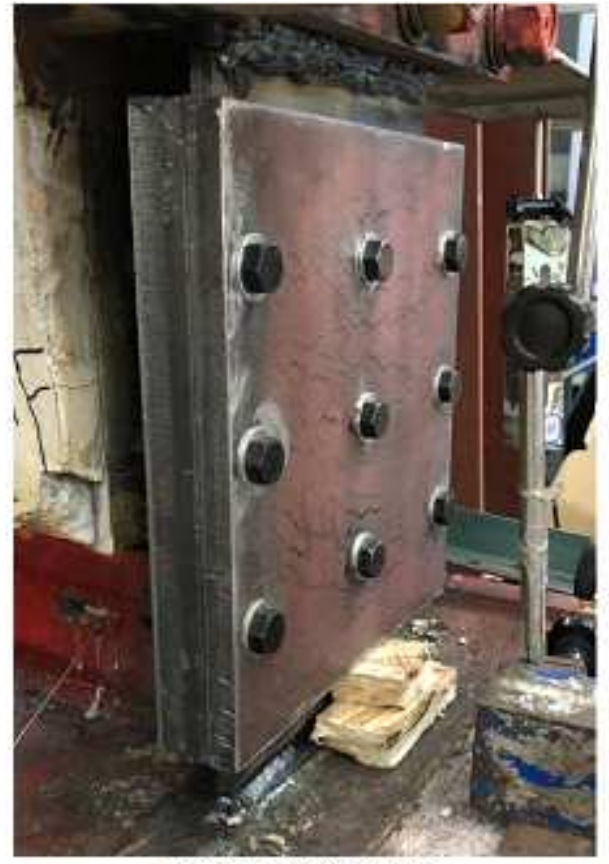

(b) Test $2(10 \mathrm{~mm})$

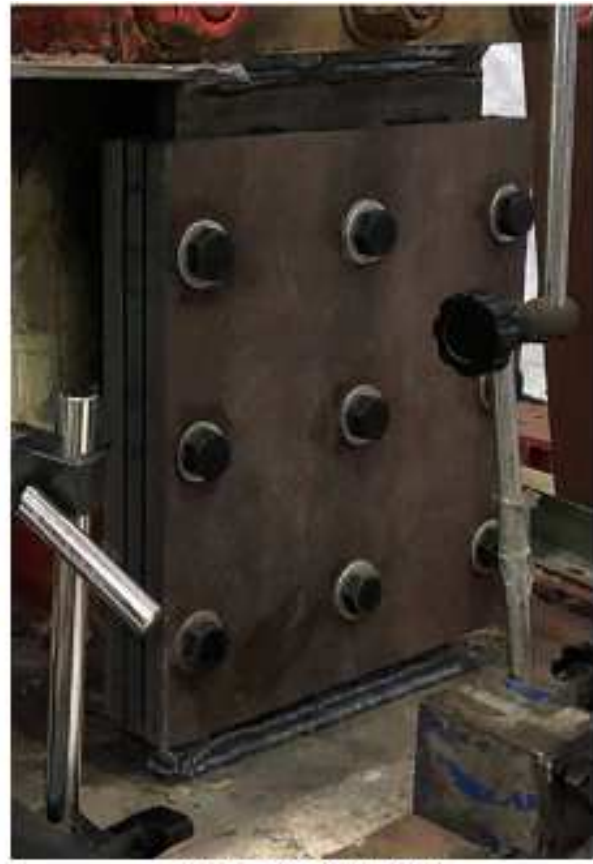

(c) Test 3 (14 mm)

Figure 10

Buckling restraint state of the REDC 


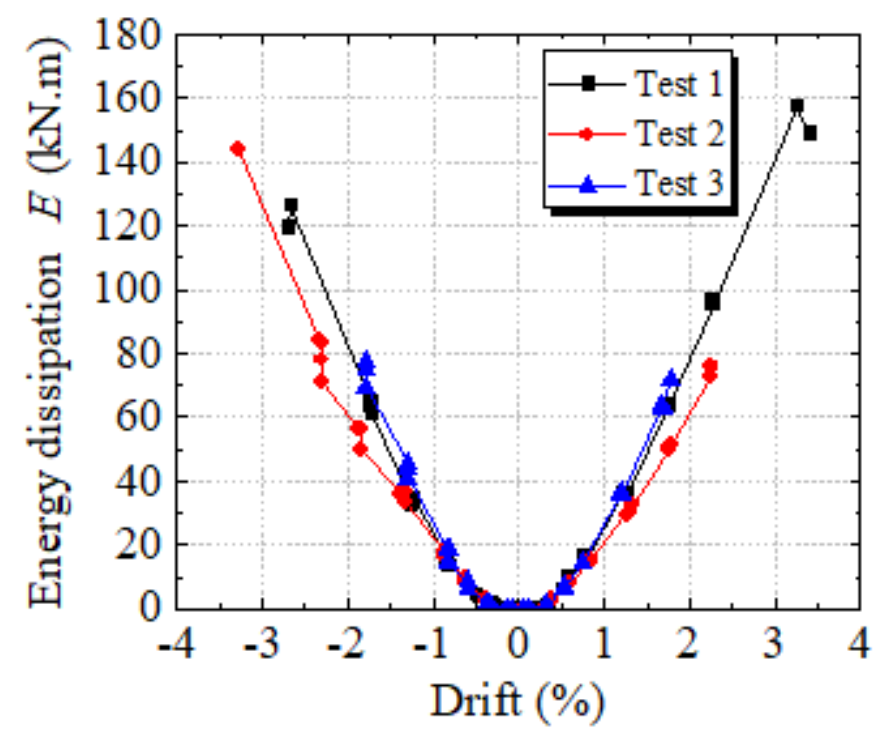

Figure 11

Energy dissipation per cycle versus drift ratio.

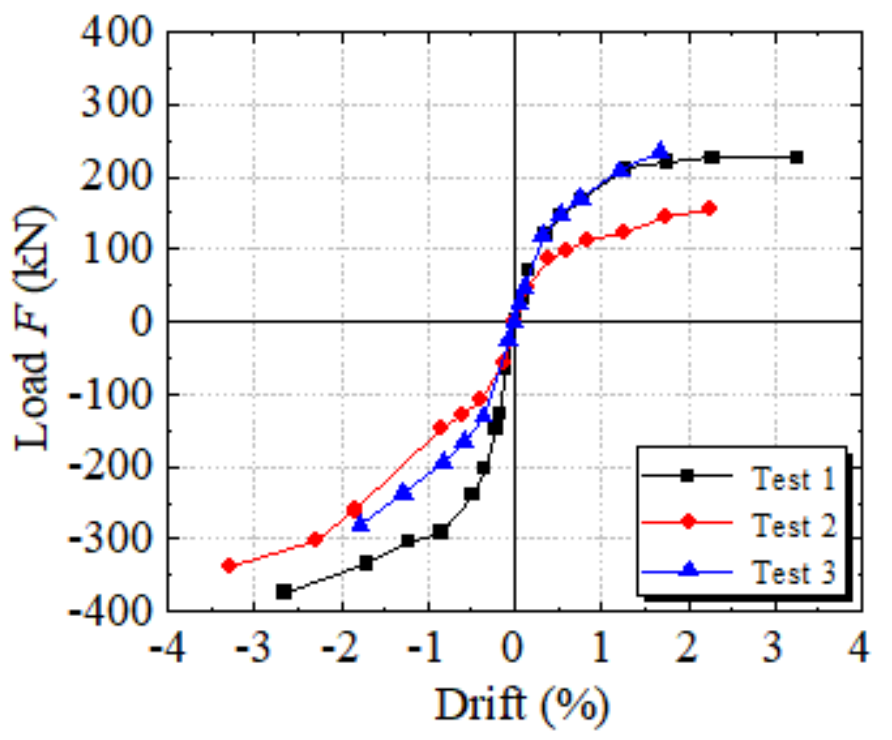

Figure 12

Skeleton curve 


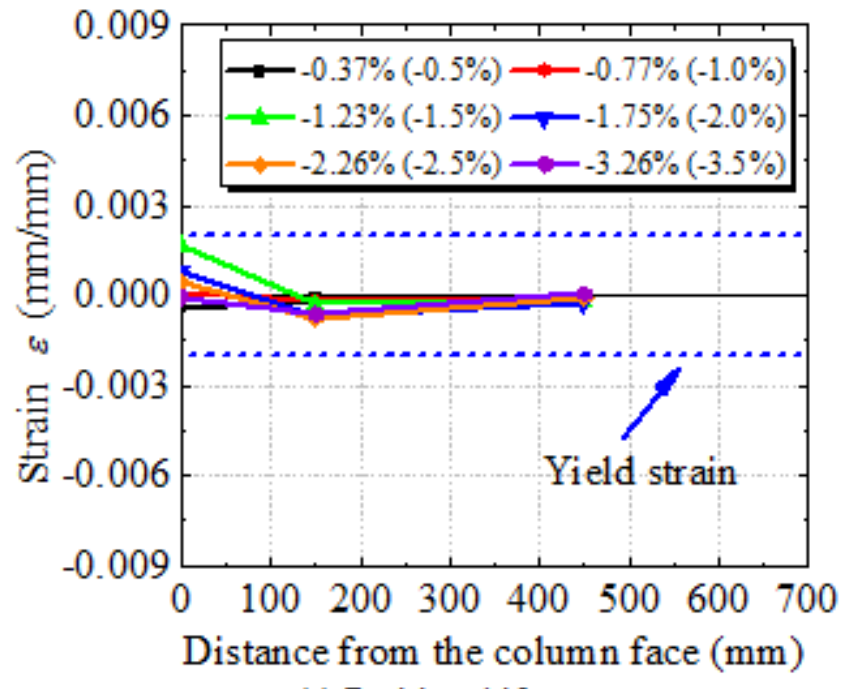

(a) Positive drifts

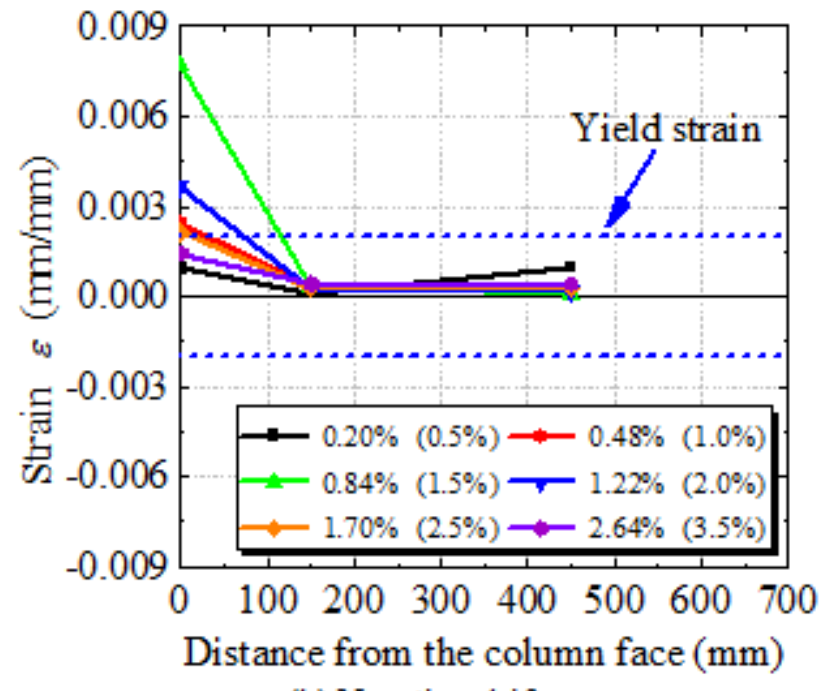

(b) Negative drifts

Figure 13

Strain profiles of the top reinforcement in Test 1

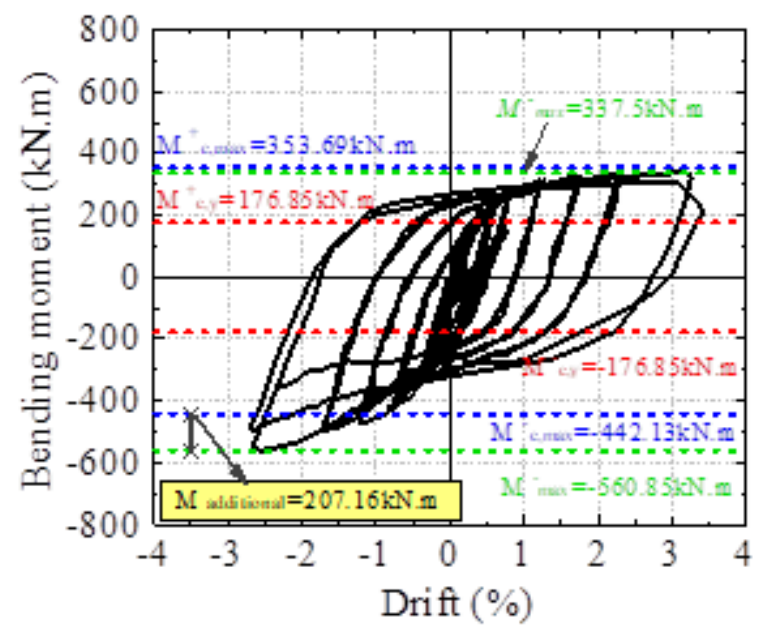

Figure 14

Schematic diagram of additional bending moment. 


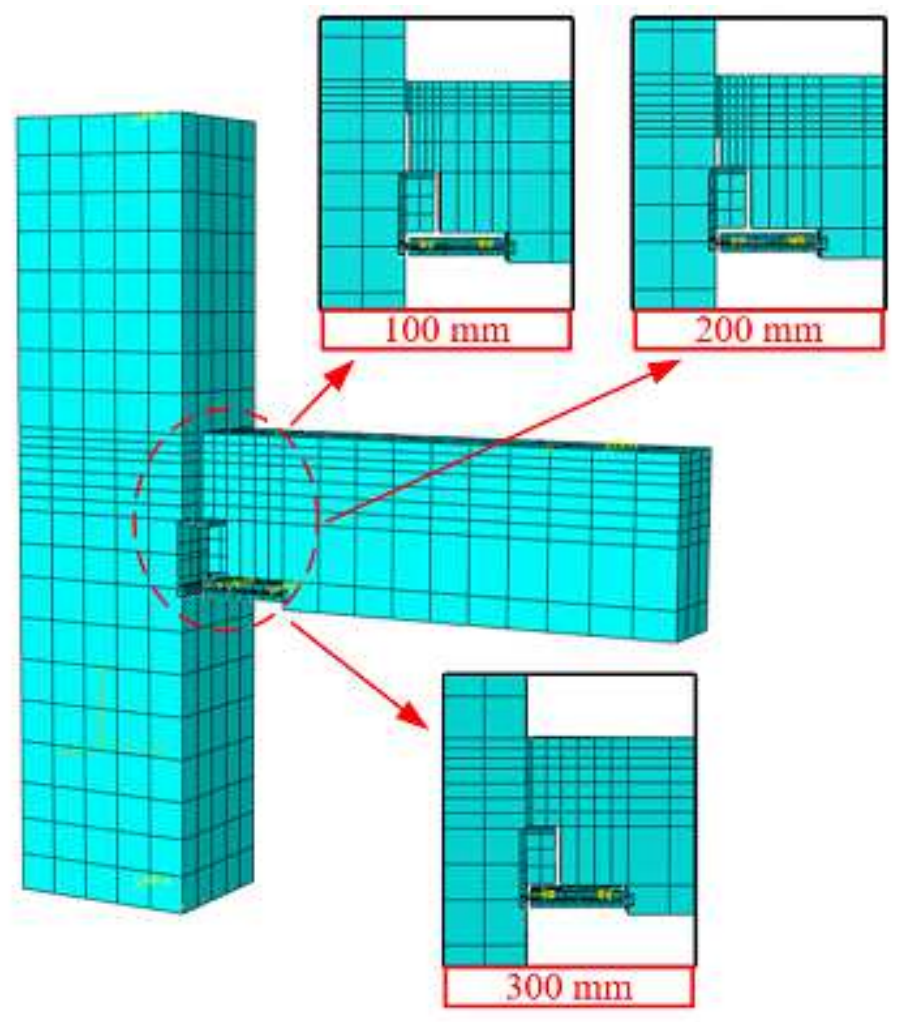

Figure 15

ABAQUS model for the novel precast connection

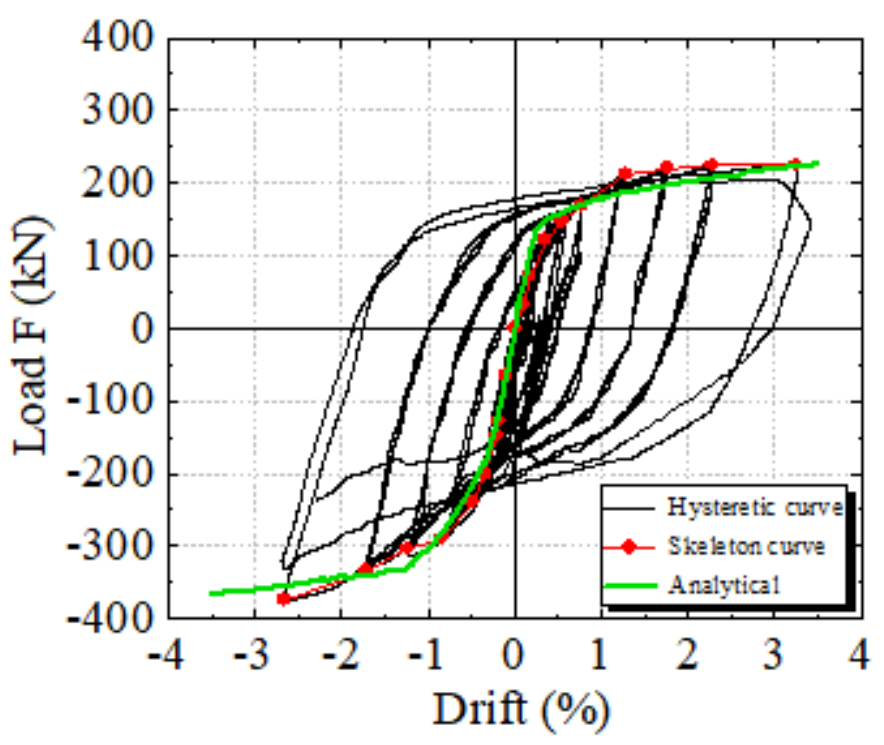

Figure 16

Comparison of analytical and experimental results 


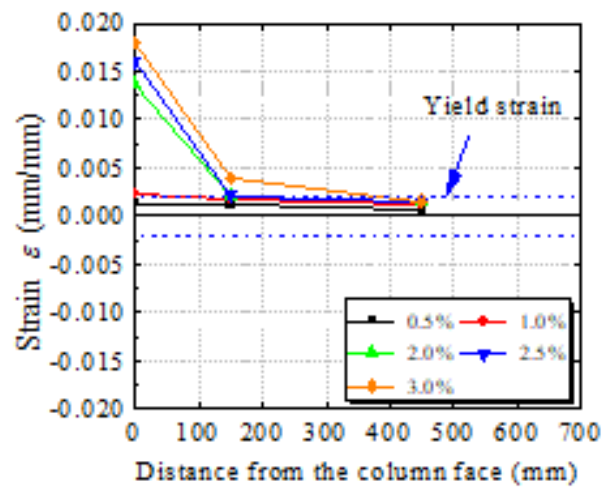

(a) Strain profile in top reinforcement at the negative peak drifts $(300 \mathrm{~mm})$

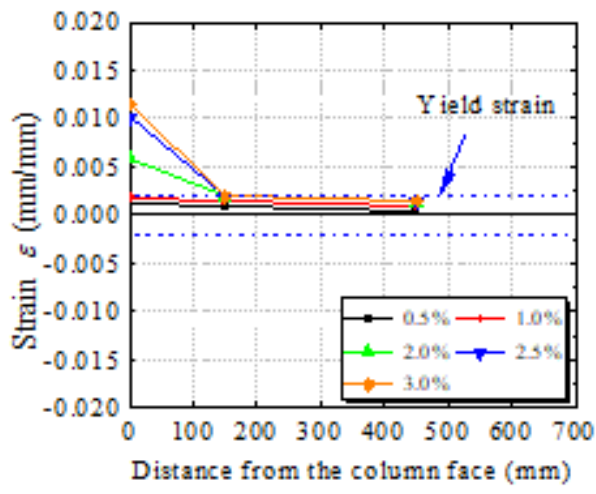

(b) Strain profile in top reinforcement at the negative peak drifts $(200 \mathrm{~mm})$

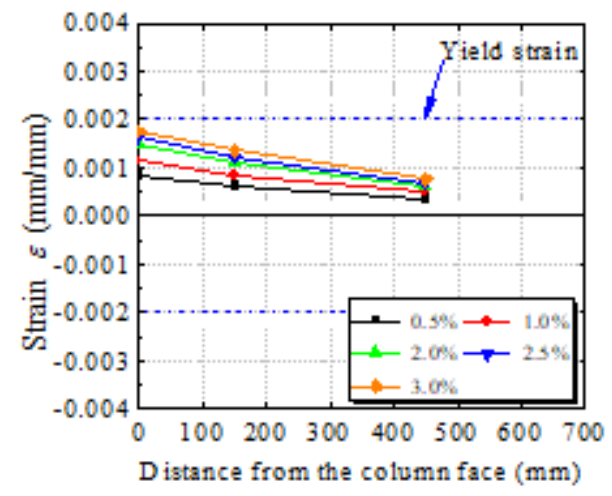

(c) Strain profile in top reinforcement at th negative peak drifts $(100 \mathrm{~mm})$

\section{Figure 17}

The peak strain of the top longitudinal reinforcement corresponding to each positive peak drift in the three models

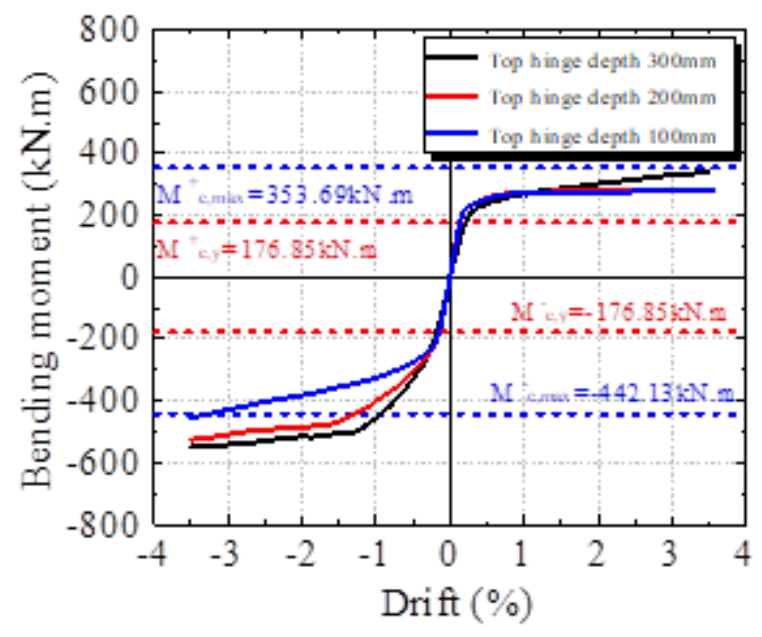

\section{Figure 18}

The comparison of the bending moment 


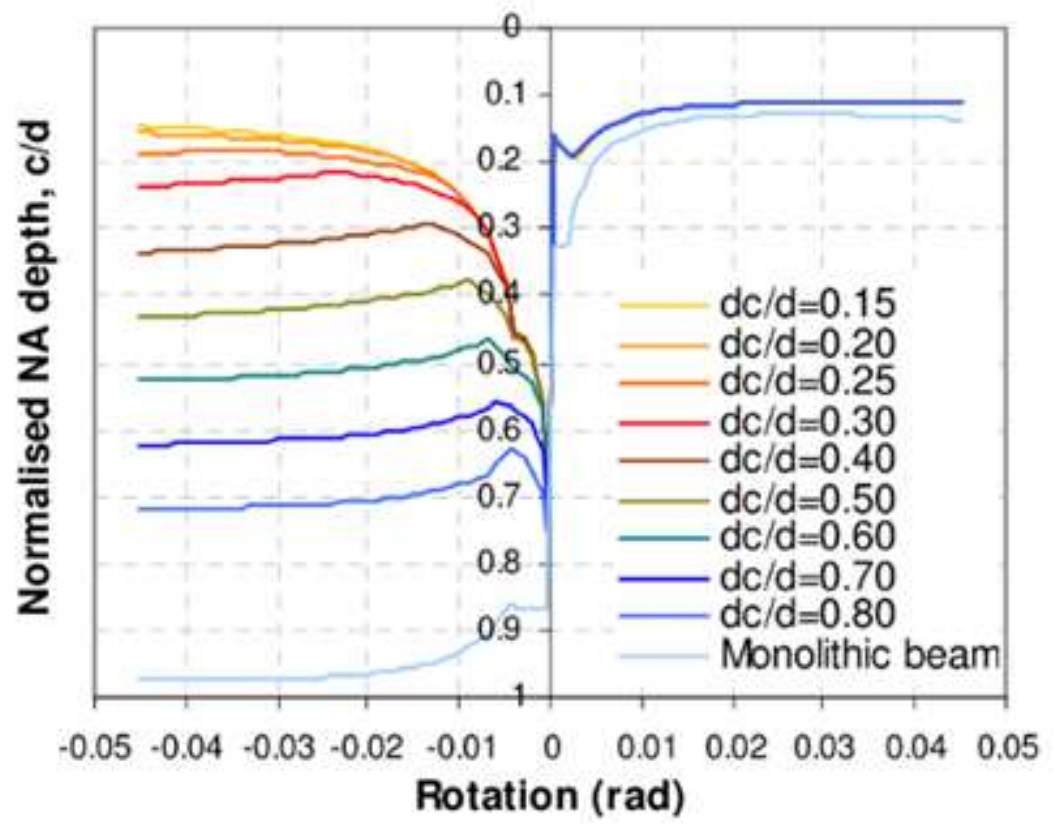

Figure 19

Parametric study for the concrete top-hinge depth (Au 2010) 